\title{
Monocytic Infiltrates Contribute to Autistic-like Behaviors in a Two-Hit Model of Neurodevelopmental Defects
}

\author{
Hong-Ru Chen, ${ }^{1}$ Ching-Wen Chen, ${ }^{1}$ Nandita Mandhani, ${ }^{2}$ Jonah C. Short-Miller, ${ }^{1}$ Marchelle R. Smucker, ${ }^{1}$ \\ Yu-Yo Sun, ${ }^{1}$ and Chia-Yi Kuan ${ }^{1,2}$ \\ ${ }^{1}$ Department of Neuroscience, Center for Brain Immunology and Glia, University of Virginia School of Medicine, Charlottesville, Virginia 22908, \\ and ${ }^{2}$ Department of Pediatrics, Emory University School of Medicine, Atlanta, Georgia 30322
}

Growing evidence suggests that early-life interactions among genetic, immune, and environment factors may modulate neurodevelopment and cause psycho-cognitive deficits. Maternal immune activation (MIA) induces autism-like behaviors in offspring, but how it interplays with perinatal brain injury (especially birth asphyxia or hypoxia ischemia [HI]) is unclear. Herein we compared the effects of MIA (injection of poly[I:C] to dam at gestational day 12.5), HI at postnatal day 10, and the combined MIA/HI insult in murine offspring of both sexes. We found that MIA induced autistic-like behaviors without microglial activation but amplified post-HI NF $\mathrm{B}$ s signaling, pro-inflammatory responses, and brain injury in offspring. Conversely, HI neither provoked autistic-like behaviors nor concealed them in the MIA offspring. Instead, the dual MIA/HI insult added autistic-like behaviors with diminished synaptic density and reduction of autism-related PSD-95 and Homer-1 in the hippocampus, which were missing in the singular MIA or HI insult. Further, the dual MIA/HI insult enhanced the brain influx of Otx2-positive monocytes that are associated with an increase of perineuronal net-enwrapped parvalbumin neurons. Using CCR2-CreER mice to distinguish monocytes from the resident microglia, we found that the monocytic infiltrates gradually adopted a ramified morphology and expressed the microglial signature genes (Tmem119, P2RY12, and Sall1) in postMIA/HI brains, with some continuing to express the proinflammatory cytokine TNF $\alpha$. Finally, genetic or pharmacological obstruction of monocytic influx significantly reduced perineuronal net-enwrapped parvalbumin neurons and autistic-like behaviors in MIA/HI offspring. Together, these results suggest a pathologic role of monocytes in the two-hit (immune plus neonatal HI) model of neurodevelopmental defects.

Key words: autism; hypoxia ischemia; maternal immune activation; monocytes; neurodevelopmental defects; neuroimmune interactions

Significance Statement

In autism spectrum disorders (ASDs), prenatal infection or maternal immune activation (MIA) may act as a primer for multiple genetic and environmental factors to impair neurodevelopment. This study examined whether MIA cooperates with neonatal cerebral hypoxia ischemia to promote ASD-like aberrations in mice using a novel two-hit model. It was shown that the combination of MIA and neonatal hypoxia ischemia produces autistic-like behaviors in the offspring, and has synergistic effects in inducing neuroinflammation, monocytic infiltrates, synaptic defects, and perineuronal nets. Furthermore, genetic or pharmacological intervention of the MCP1-CCR2 chemoattractant pathway markedly reduced monocytic infiltrates, perineuronal nets, and autistic-like behaviors. These results suggest reciprocal escalation of immune and neonatal brain injury in a subset of ASD that may benefit from monocyte-targeted treatments.

Received May 13, 2020; revised 0ct. 19, 2020; accepted 0ct. 22, 2020.

Author contributions: H.-R.C. and C.-Y.K. designed research; H.-R.C., C.-W.C., N.M., J.C.S.-M., M.R.S., and

Y.-Y.S. performed research; H.-R.C., C.-W.C., and N.M. analyzed data; H.-R.C. wrote the first draft of the paper;

C.-Y.K. edited the paper; C.-Y.K. wrote the paper.

The authors declare no competing financial interests.

This work was supported by the National Institute of Health Grants NS095064, NS100419, and NS108763 to C.-Y.K. and NS106592 to Y.-Y.S., and American Heart Association Postdoctoral Fellowship 18P0ST34080334 to H.-R.C.

Correspondence should be addressed to Chia-Yi Kuan at alex.kuan@virginia.edu.

https://doi.org/10.1523/JNEUROSCI.1171-20.2020

Copyright $\odot 2020$ the authors

\section{Introduction}

Autism spectrum disorder (ASD) is a heterogeneous group of pervasive developmental disorders with persistent deficits in social communication and social interactions across multiple contexts (American Psychiatric Association, 2013). Likewise, the pathogenesis of ASD involves complex interactions of genetic, immune, and environmental factors (Geschwind, 2009; De Rubeis et al., 2014; Estes and McAllister, 2016). Among these three categories of ASD risk factors, prenatal infection or maternal immune activation (MIA) is common, but not always 
coupled with neurodevelopmental defects. Hence, MIA may function as a primer for an array of genetic and environmental factors to impair neurodevelopment (Meyer, 2014). Consistent with this notion, MIA has been shown to sensitize schizophrenia-related behaviors in mice with peripubertal stress or the Disc1 mutation (Giovanoli et al., 2013; Lipina et al., 2013). Yet, whether and how MIA interacts with perinatal hypoxia ischemia (HI) to enhance autistic-like behaviors remain uncertain.

Indirect evidence suggested that perinatal asphyxia and $\mathrm{HI}$ may be a contributing factor to ASD (van Handel et al., 2007). Even in the landmark study of genetic influences in autism, the researchers already noticed a higher incidence of autism in twins with perinatal apnea $>6$ min or $>30$ min delay in the second birth, which could produce cerebral HI (Folstein and Rutter, 1977). Another study reported $5 \%$ versus $0.8 \%$ diagnosis of ASD at 5 years of age in term infants with or without perinatal $\mathrm{HI}$, respectively (Badawi et al., 2006). Last, a recent epidemiological study of 594,638 subjects showed a significant association between ASD and perinatal HI symptoms, including birth asphyxia and Apgar score $<7$ at $5 \mathrm{~min}$ after birth (Getahun et al., 2017). Together, these observations suggest that MIA and cerebral HI may cooperate to induce autistic behaviors, but the underlying mechanisms are poorly understood.

In this study, we injected polyinosinic:polycytidylic acid (poly (I:C)) to pregnant C57BL/6 mice at gestational day 12.5 and challenged the offspring with $\mathrm{HI}$ at postnatal day 10 as a two-hit MIA/HI model, and compared the outcomes with those after MIA or pure-HI insult. In addition to autistic-like symptoms (Malkova et al., 2012), we compared the effects of MIA, HI, and dual-MIA/HI on synaptic density, microglial activation, monocytic infiltrates, and perineuronal nets (PNNs) following several leads in the literature. First, many ASD mutations converge on the synaptic genes, but MIA-alone only modestly impaired synaptic maturation (Coiro et al., 2015; Bergdolt and Dunaevsky, 2019). Thus, we tested whether the combined MIA/HI insult is more hurtful to synaptic density and autism-related synaptic proteins, such as PSD-95 and Homer-1 (Tsai et al., 2012; Lelieveld et al., 2016). Second, microglial activation has been detected in ASD patients (Morgan et al., 2010; Suzuki et al., 2013), but to what extent this phenomenon is captured by the rodent MIA model is uncertain (Smolders et al., 2015; Choi et al., 2016). Will the combination of MIA and HI activate microglia? Third, mice with early-life adversity or the Rett syndrome mutation contain more PNNs, the extracellular matrix assemblies that preferentially enwrap parvalbumin (PV)-positive interneurons and may impair synaptic plasticity (Krishnan et al., 2015; Fawcett et al., 2019; Murthy et al., 2019). Thus, we tested whether MIA or MIA/HI alters PNNs in offspring correlated with autistic-like behaviors. Finally, because an increase of immune infiltrates has been detected in the brains of ASD patients (DiStasio et al., 2019), we conjectured that the dual MIA/HI insult may boost immune infiltrates, including monocytes that have the potential to become proinflammatory microglia to damage the synaptic network (Geissmann et al., 2003; Djukic et al., 2006; Schafer et al., 2012; Wu et al., 2015; Cronk et al., 2018; Chen et al., 2020).

The results of our experiments showed that MIA and neonatal HI injury have synergistic effects in inciting neuroinflammation, monocytic infiltration, PNNs, and synaptic defects, while preserving the autistic-like behaviors in murine offspring. Blocking monocyte infiltration via the MCP1-CCR2 signaling pathway markedly reduced the PNN intensity and autistic-like behaviors in offspring. Together, these results suggest immune and brain injury interactions in a subset of ASD, which may benefit from monocyte-targeted treatment.

\section{Materials and Methods}

Animals and experimental design. C57BL/6J mice, $C X 3 C R 1^{G F P}$ mice (JAX\#005582), CCR2 $2^{R F P}$ (JAX\#017586) mice, and R26R-TRAP (translating ribosome affinity purification, JAX stock \#022367) mice were purchased from The Jackson Laboratory. CX3CR1 $1^{G F P}$; CCR2 $2^{R F P}$ mice were derived from breeding $C X 3 C R 1^{G F P}$ mice with $C C R 2^{R F P}$ mice. The CCR2-CreER(T2) mice have been characterized recently (Chen et al., 2020). The CCR2-CreER mice were crossed with a R26R-GFP Cre-reporter line (Ai6, JAX stock \#007906) or R26R-TRAP (GFP/RpL10A) mice (JAX stock \#022367). For fate-mapping, CCR2-CreER mice crossed to either R26R-GFP or R26R-GFP/RpL10A TRAP mice received $10 \mathrm{mg} /$ $\mathrm{kg} /$ day tamoxifen at P8 and P9. Mice were housed in ventilated cages under standard laboratory conditions.

For the timed pregnancies, mice were set up in the late afternoon and plugs were detected the next morning, the stage of embryogenesis designated as E0.5. At E12.5, pregnant dams received intraperitoneal injection of one dose of $20 \mathrm{mg} / \mathrm{kg}$ poly(I:C) (Sigma Millipore, \#P9852), a synthetic analog of double-stranded RNA to activate the Toll-like receptor 3 (Stridh et al., 2013). Poly(I:C) was freshly dissolved in saline. The dose of poly(I:C) and its route of drug-delivery were selected based on previous studies (Choi et al., 2016). Dams were monitored for parturition, and the offspring of both sexes were used in experiments and analyzed separately in brain tissue loss and behavioral test. To avoid litterassociated bias, mice from at least three litters were used in all analyses.

HI surgery and LPS treatment. The pups of indicated group were collected and subjected to the Rice-Vannucci model of HI (unilateral common carotid artery ligation followed by $60 \mathrm{~min}$ exposure to $10 \%$ oxygen at $37^{\circ} \mathrm{C}$ ) at $10 \mathrm{~d}$ of age. The procedures of $\mathrm{HI}$ and LPS-sensitized $\mathrm{HI}$ have been described previously (Rice et al., 1981; Eklind et al., 2005; Adhami et al., 2008; Stridh et al., 2013; Yang et al., 2013). The controls include age-matched sham and MIA- or HI-alone injury. For the LPS/HI injury, used solely as positive controls for NF $\kappa$ B signaling activation, $0.3 \mathrm{mg} / \mathrm{kg}$ LPS (Sigma Millipore, \#L2880, from Escherichia coli 005:B5) was injected intraperitoneally to P10 mouse pups $2 \mathrm{~h}$ before the HI procedures. All experimental procedures were approved by the Institutional Animal Care and Use Committee and conformed to the National Institutes of Health Guide for the care and use of laboratory animals.

TUNEL assay. The $20 \mu \mathrm{m}$ cryosections were cut from the brains of littermates transcardially perfused with $4 \%$ PFA, postfixed with gentle agitation at $4^{\circ} \mathrm{C}$ overnight, dehydrated in $30 \%$ sucrose, followed by freezing and sectioning. Sections were stored at $-20^{\circ} \mathrm{C}$ until the TUNEL assay was performed. TUNEL staining was used to identify apoptotic cells under light microscope using the Click-iT TUNEL AlexaFluor-647 Imaging Assay kit (Thermo Fisher Scientific) following the manufacturer's instructions.

Measurement of brain tissue loss. Brain tissue loss was performed at $7 \mathrm{~d}$ after MIA, pure-HI, or dual MIA/HI injury, as previously described (Yang et al., 2013). Briefly, animals were killed under deep anesthesia by transcardiac perfusion of saline, and the brains were fixed in $4 \%$ PFA overnight, followed by serial sectioning of $1 \mathrm{~mm}$ slices on a Vibratome (Stoelting). Each section was photographed, and the tissue loss in the cerebral cortex, striatum, and the hippocampus was quantified as the area percentage of all sections containing the structure of interest on the ipsilateral to contralateral hemisphere by the National Institutes of Health ImageJ software.

Protein extraction and Western blotting. The indicated mouse brain tissues were isolated to compare the $\mathrm{NF} \kappa \mathrm{B}$ signaling activity. The extraction of the nuclear fraction and cytoplasmic protein was performed using the NE-PER Nuclear and Cytoplasmic Extraction Reagents (Thermo Fisher Scientific) according to the manufacturer's instructions. Brain nuclear fraction, cytoplasmic protein extracts, or total protein lysate were separated by the standard SDS-PAGE procedure, electrotransferred to a microporous PVDF membrane (Bio-Rad), and detected with designated antibodies using the HRP chemiluminescence reagents (GE Healthcare). The following antibodies were used: mouse anti-NF $\kappa \mathrm{B}$ 
inhibitor (Cell Signaling Technology); rabbit anti-phoshpo-NF- $\kappa \mathrm{B} / \mathrm{p} 65$ (Ser276) (Cell Signaling Technology); rabbit anti-Histone 2B (Cell Signaling Technology); rabbit anti-GAPDH (Cell Signaling Technology); rabbit antiSynaptotagmin 1 (Synaptic Systems); mouse anti-PSD-95 (Millipore); rabbit anti-Homer 1 (Abcam); and rabbit anti-MeCP2 (Abcam).

$R N A$ extraction and $q R T-P C R$. The total RNA of the indicated brain samples was extracted using the TRIzol reagent (Thermo Fisher Scientific); $3 \mu \mathrm{g}$ RNA was used for cDNA synthesis using High-Capacity cDNA Reverse Transcription Kit (Applied Biosystems) according to the manufacturer's instructions. qRT-PCR was performed using the BioRad CFX 96 system (C1000 Thermal Cycler) and detected by SYBR Green master mix (Bio-Rad) as previously described (Yang et al., 2013). The following primer sequences were used for real time PCR: $I L-6,5^{\prime}$ GGAGAGGAGACTTCACAGAGGAT-3' and $5^{\prime}$-AGTGCATCATCG CTGTTCATA; C-3'; $I L-17 \alpha, 5^{\prime}$-AGACTACCTCAACCGTTCCACTT$3^{\prime}$ and $5^{\prime}$-GATCTCTTGCTGGATGAGAACAG- $3^{\prime}$; $I L-1 \beta, 5^{\prime}$-CTTT CGACAGTGAGGAGAATGAC- ${ }^{\prime}$ and $5^{\prime}$-CAAGACATAGGTAGCT GCCACAG-3'; MMP9, 5' -CGAACACCACGCTGTGGTG-3' and 5'CCGCTTCGGGTCCGTACAC-3'; TNF $\alpha, 5^{\prime}$-CCACCACGCTCTTCT GTCTA-3' and 5'-CTCCTCCACTTGGTGGTTTG-3'; IL-23, 5' - AC CAGTGGGACAAATGGATCTAC- $3^{\prime}$ and $5^{\prime}$-CAGGTGCTTATAAA ACACCAGACC-3'; TSPO, 5' -CTATGGTTCCCTTGGGTCTCTAC-3' and 5'-AGGCCAGGTAAGGATACAGCAAG-3'; $M C P 1$, 5' - ACCACT ATGCAGGTCTCTGTCAC-3' -and 5' -GCTGCTGGTGATTCTCTTGT AGT-3'; OPN, 5' -AGCCACAAGTTTCACAGCCACAAGG-3' and 5'CTGAGAAATGAGCAGTTAGTATTCCTGC-3'; CCR2, 5' -GCCAGG ACAGTTACCTTTGG-3' and 5' - CGAAACAGGGTGTGGAGAAT-3'; Sall 1, 5'-CTCAACATTTCCAATCCGACCC-3' and 5'- GGCATCCT TGCTCTTAGTGGG-3'; P2RY12, 5' -TTTCAGATCCGCAGTAAATC CAA-3' and 5' - GGCTCCCAGTTTAGCATCACTA-3'; Tmem119, 5' CCTACTCTGTGTCACTCCCG- $3^{\prime}$ and $5^{\prime}$-CACGTACTGCCGGAAG AAATC-3'; Otx2, 5' - TATCTAAAGCAACCGCCTTACG-3' and $5^{\prime}-$ GCCCTAGTAAATGTCGTCCTCTC-3'; Otx1, 5' - GCGCTGTTCGC AAAGACTC-3' and 5'-GTTTTCGTTCCATTCCCGTC-3'; GAPDH, $5^{\prime}$-AGGTCGGTGTGAACGGAT TTG-3' and $5^{\prime}$-TGTAGACCATG TAGTTGAGGTCA-3'. Samples were analyzed in triplicate and normalized versus the expression level of the GAPDH.

Ultrasonic vocalization recording. On postnatal days 9-11, the cages containing both mothers and litters were brought to the testing room. After mice are habituated for $1 \mathrm{~h}$, pups were removed away from the mother, and put into a cup with bedding from their cage for $10 \mathrm{~min}$ without recording. After the $10 \mathrm{~m}$ habituation period, mice were individually placed in a clean holding dish. The ultrasonic vocalization (USV) by pups was recorded for $3 \mathrm{~min}$ using an ultrasound-grade microphone (Pettersson M500-384 USB, Pettersson Elektronik). The recording was analyzed with the UltraVox software (Noldus Information Technology). The USV s between 25 and $125 \mathrm{kHz}$ were analyzed after excluding the background noise that was $<0.02 \mathrm{~ms}$ (Lammert and Lukens, 2019).

Three chamber social preference. Eight-week-old mice (in the C57BL/6 strain background) were assessed for social preference using the three-chamber social approach. This sociability apparatus consists of an acrylic box partitioned into three chambers (the overall dimensions: 24 inch wide $\times 16$ inch deep $\times 8.5$ inch tall). The openings between the compartments allow the animals to freely explore the three chambers. During an initial moment, an inanimate object was positioned in one of the lateral chambers, and a novel mouse was placed in the opposite lateral chamber. This novel mouse was sex-matched and of no previous contact with the tested animal. The time spent in each chamber, as well as the time spent exploring the novel mouse or the inanimate object, was analyzed by two observers during $10 \mathrm{~min}$. We evaluated the sociability index (SI), a mathematical equation designed to allow direct comparison of social behavior of the groups (Pietropaolo et al., 2011). The SI was calculated as follows: $\mathrm{SI}=$ (time exploring novel mouse $) /($ time exploring novel mouse + time exploring novel object). The time exploring each chamber by the tested animal was also collected to exclude the difference in overall locomotion activity.

Marble burying test. Eight-week-old mice (in the C57BL/6 strain background) were acclimated overnight to cages containing the wood chip bedding to minimize neophobia and novelty-induced anxiety. The next day, the mice were placed in a clean cage that was filled with 2 inches of the wood chip bedding. Twenty glass marbles were arranged on the top of the bedding in five rows of four marbles equidistant from one another. After a 15 min exploration period, the tested mouse was carefully removed from the cages; and a marble burying index score was calculated based on the following scale: 1 for marbles covered $50 \%$ by bedding, 0.5 for $\sim 50 \%$ covered, or 0 for anything less. After each testing period, the marbles were cleaned with $70 \%$ ethanol. All procedures were essentially the same as previously described (Lammert and Lukens, 2019).

Flow cytometry analysis. To analyze the infiltrating immune cells in the brains after MIA, HI, and MIA/HI, the mice were transcardially perfused with PBS to remove blood cells in cerebral vessels. Sham and MIA offspring brains and the ipsilateral hemispheres at $96 \mathrm{~h}$ after HI or MIA/ HI were harvested. The brain tissues were mechanically homogenized, and the infiltrating immune cells were isolated by discontinued Percoll gradients (GE Healthcare). Cell suspensions were stained with the fluorochrome-conjugated antibodies, including CD45 (30-F11, BioLegend), CD11b (M1/70, BioLegend), Ly-6G (1A8, BD Bioscience), Ly-6C (HK1.4, BioLegend), CD3 (17A2, BioLegend), Trem2 (clone \#237920, R\&D Systems), $\gamma \delta$ TCR (GL3, BioLegend), and LIVE/DEAD Fixable Aqua Dead Cell Stain (Thermo Fisher Scientific). Subsequently, the stained suspensions were collected with flow cytometric analyses (Attune, Applied Biosystems) and analyzed with FlowJo v10.

Immunohistochemistry and Wisteria floribunda agglutinin (WFA) staining. The brains from indicated littermates were fixed using 4\% PFA at $4^{\circ} \mathrm{C}$ overnight with gentle agitation, cryopreserved in $30 \%$ sucrose, frozen, and stored at $-20^{\circ} \mathrm{C}$ until use. For staining, $20 \mu \mathrm{m}$ cryosections were made and incubated in blocking/permeabilization solution containing 3\% NGS and $0.2 \%$ Triton-X in PBS. The sections were treated overnight with appropriate primary antibodies or fluorescein-conjugated WFA (1:500, Vector Laboratories) diluted in $1 \%$ NGS/0.2\% Triton X100/PBS, followed by species-specific secondary antibodies for $2 \mathrm{~h}$ at room temperature. The following primary antibodies were used: rabbit anti- CD68 (Abcam); rat anti-C3 (Abcam); rabbit anti-NeuN (Abcam); rabbit anti-Synaptotagmin 1 (Synaptic Systems); mouse anti-PSD-95 (Millipore); rabbit anti- TNF $\alpha$ (clone 2C8, Abcam ab8348); rabbit anti-Iba1 (Wako Chemicals); rabbit anti-Tmem119 (Abcam); rat anti-P2RY12 (BioLegend); mouse anti-PV $(\sigma)$; and rabbit anti-Otx2 (Abcam).

Quantification of synaptic density and cell number. Images were acquired using a SP8 confocal microscope with $20 \times$ magnification with an image matrix of $1024 \times 1024$ pixel, a pixel scaling of $0.2 \times 0.2 \mu \mathrm{m}$ and a depth of 8 bit. Confocal images were collected in $z$ stacks with a slice distance of $0.4 \mu \mathrm{m}$. Analyses of immunostaining and cell quantification were performed using the National Institutes of Health ImageJ software. The synaptic density was measured by confocal microscopy analysis of anti-synaptotagmin, anti-PSD-95, and colocalized signals. To standardize the imaging analysis, a $z$ stack of optical sections from the hippocampal CA3 region was captured from each section, then were counted in each $10 \mu \mathrm{m}^{2}$ square. The measurements from 10 randomly selected squares were collected in each division for statistical analysis. For cell number comparison, the measurements were made from 5 randomly selected $300 \mu \mathrm{m}^{2}$ squares.

RNAscope assay. Mouse brain was freshly perfused and fixed in $4 \%$ PFA for $24 \mathrm{~h}$ at $4^{\circ} \mathrm{C}$. One day later, we immersed the brain in $30 \%$ sucrose, allowing the tissue to sink to the bottom of the container. Brain tissue was frozen on dry ice in the Optimal Cutting Temperature embedding media, and cut into $15 \mu \mathrm{m}$ sections. The RNA probes were purchased from Advanced Cell Diagnostics. The probes against mouse Sall1 (ACD, catalog \#469661-C3), positive control (ACD, catalog \#310771), and negative probe (ACD, catalog \#310043) were used. ISH was performed using the RNAscope Flurorescent Multiplex Reagent Kit (ACD, catalog \#320850) according to the manufacturer's instructions and as described previously (Wang et al., 2012).

Purification of cell type-specific mRNA from CCR2-CreER; R26RTRAP mice. Bone marrow, peripheral blood, and dissected mouse brains were immediately homogenized in ice-cold dissection buffer following the previously described TRAP procedures (Heiman et al., 2014). 
Drug administration. The P10 pups were injected intraperitoneally with RS102895 (20 mg/kg \#R1903, dissolved in 20\% 2-hydroxypropyl$\beta$-cyclodextrin solution, Sigma Millipore, \#H107), which is a specific CCR2-receptor antagonist (Mirzadegan et al., 2000), or vehicle immediately after the MIA/HI insult.

Statistical analyses. Data are presented as the mean \pm SEM. Statistical analysis was performed using GraphPad Prism version 7.0 (GraphPad Software). Comparison between two groups was performed by Student's $t$ test. Comparison between four groups (UN, MIA, HI, and MIA/HI) of both sexes was performed by two-way ANOVA followed by Tukey's multiple comparisons post hoc test for behavior tests and tissue loss results. Differences between four groups (UN, MIA, HI, and MIA/ $\mathrm{HI}$ ) were analyzed by one-way ANOVA followed by Tukey's multiple comparisons post hoc test without considering the gender effect for most results. For sample size $\leq 4$, a Kruskal-Wallis $(\mathrm{H})$ test followed by Dunn's multiple comparison test was used. A $p$ value $<0.05$ was considered statistically significant. Specific statistical tests used were specified in the corresponding figure legends.

\section{Results}

\section{MIA amplifies NF $\kappa \mathrm{B}$ signaling and inflammatory responses} to neonatal cerebral $\mathrm{HI}$

We first examined whether prenatal MIA (at E12.5) primed the offspring to mount more severe inflammatory responses to cerebral $\mathrm{HI}$ at $\mathrm{P} 10$, which is $18 \mathrm{~d}$ apart (Fig. $1 \mathrm{~A}$ ). When probed by immunoblots at P11 (i.e., $24 \mathrm{~h}$ after HI, MIA/HI, or LPS-sensitized HI), the MIA offspring and the ipsilateral cortex of post-HI neonates exhibited a small increase of nuclear phosphorylated $\mathrm{NF} \kappa \mathrm{B} / \mathrm{p} 65$ and an inverse reduction of cytoplasmic $\mathrm{I} \kappa \mathrm{B} \alpha$, in great contrast to marked responses in the MIA/HI or LPS/HIinjured brains (Fig. $1 B, C ; n=3$ from three litters for each group; Kruskal-Wallis test, $p<0.0001$; mean $\pm \mathrm{SEM}, \mathrm{UN}=1 \pm 0.31$, $\mathrm{MIA}=3.21 \pm 0.62, \quad \mathrm{HI}=2.76 \pm 0.67, \quad \mathrm{MIA} / \mathrm{HI}=11.66 \pm 0.88$, $\mathrm{LPS} / \mathrm{HI}=15.33 \pm 0.87$; the LPS/HI-injured brains were used as a positive control for $\mathrm{NF} \kappa \mathrm{B}$ induction). The MIA offspring brains also showed a small, but significant, increase of $I L-6, I L-17 \alpha$, and MMP9 mRNAs, but not the other examined proinflammatory cytokines, in P11 offspring brains (Fig. $1 D ; n=6$ each for naive and MIA). These results support a causal role for IL-6 and IL$17 \alpha$ in MIA, as suggested previously (Smith et al., 2007; Choi et al., 2016).

In contrast, the dual MIA/HI insult markedly elevated the mRNA levels for all examined proinflammatory cytokines, including $I L-6, I L-17 \alpha, T N F \alpha, M C P-1, I L-23, M M P 9$, TSPO (translocator protein, a marker of microglial activation), and OPN (osteopontin, another marker for macrophages/activated microglia) compared with naive neonates and HI-injured neonates (note the discontinuous scale of $y$ axis in Fig. $1 D ; n=6-9$ in each group; one-way ANOVA; $I L-6, F_{(3,26)}=4.27, p=0.014$; $I L-17, F_{(3,26)}=9.82, p=0.0002 ; T N F \alpha, F_{(3,26)}=4.91, p=0.0078$; $M C P-1, F_{(3,26)}=2.81, p=0.049 ;$ TSPO, $F_{(3,26)}=10.03, p=0.0001$; $I L-23, F_{(3,26)}=4.48, p=0.0115 ; M M P 9, F_{(3,26)}=3.65, p=0.025$; OPN, $\left.F_{(3,26)}=4.21, p=0.014\right)$. Further, MIA neither induced TUNEL-positive cell death nor caused discernible brain atrophy in offspring, but it increased these brain injury indicators when combined with neonatal HI (Fig. $1 E-G ; n=3$ for each group in TUNEL stain; Kruskal-Wallis test, $p=0.0003$; mean \pm SEM, $\mathrm{UN}=1 \pm 0.2, \mathrm{MIA}=1.68 \pm 0.46, \mathrm{HI}=11.92 \pm 3.36, \mathrm{MIA} / \mathrm{HI}=$ $37.87 \pm 7.51)$. Both male and female murine neonates showed greater brain damage in MIA/HI injury than pure HI injury (Fig. $1 G ; n=4-11$ for each group/gender in the brain atrophy analysis; two-way ANOVA, $F_{(2,34)}=24.62, p<0.0001$; mean \pm SEM, MIA= $4.25 \pm 2.13$, HI $=10 \pm 3.54, \mathrm{MIA} / \mathrm{HI}=47.36 \pm 6.07$; female, $\mathrm{MIA}=3 \pm 0.54, \mathrm{HI}=12.5 \pm 3.94, \mathrm{MIA} / \mathrm{HI}=36.62 \pm 7.4$ ). Together, these results suggest that prenatal MIA is a primer for more severe brain injury and inflammatory responses to cerebral $\mathrm{HI}$ in the offspring neonates.

\section{HI preserves autistic-like behaviors in MIA offspring and increases the synaptic damage}

Next, we tested whether neonatal $\mathrm{HI}$ induces or obscures the mouse versions of ASD symptoms in the MIA offspring. Consistent with a previous report (Malkova et al., 2012), prenatal MIA reduced USV by P11 pups when separated from dam and the preference for an unfamiliar mouse over a novel object in the three-chamber test (sociability), and produced a trend of more marble-burying behaviors in adults (Fig. $2 A-E ; n=7$ 21 for each group/gender; two-way ANOVA, USV, $F_{(3,125)}=$ $28.21, p<0.0001$; sociability, $F_{(3,64)}=12.41$, $p<0.0001$; distance traveled, $F_{(3,69)}=28.21, p=0.3$; repetitive behaviors, $\left.F_{(3,67)}=10.54, p<0.0001\right)$. Pure-HI decreased USV only in female P11 pups but had no effects on the sociability and repetitive behaviors in both male and female mice at 2 months of age (Fig. $2 A-E ; n=8-22$ for each test/gender). In contrast, the MIA/HI-injured offspring maintained the aberrations in USV, social preference, and repetitive behaviors similar to the MIA offspring (Fig. $2 A-E ; n=9-21$ for each test/gender). Of note, no difference in locomotor activity was noticed between experimental groups, as shown by comparable traveled distances in the three-chamber test (Fig. 2D, right).

We also quantified anti-synaptotagmin (presynaptic), antiPSD-95 (postsynaptic), and colocalized anti-synaptotagmin/PSD95 immunofluorescent puncta on confocal microscopy to compare the effects of MIA, HI, and MIA/HI on the synaptic density in P17 hippocampus (Fig. $2 F, G$ ). We focused the analysis on hippocampus because this region is rarely damaged under the severity of HI and MIA/HI insult in our studies. To compare both presynaptic and postsynaptic proteins allows us to test tissue injury and determine whether the postsynaptic machinery is more closely linked to ASD-like anomalies, as suggested by genetic studies (De Rubeis et al., 2014). This analysis indicated that only dual-MIA/HI caused significant reduction of anti-PSD-95 and anti-synaptotagmin/PSD-95 puncta (synapses), but not the presynaptic anti-synaptotagmin puncta, in the CA3 sector (Fig. $2 F, G ; n=3$ for each group; Kruskal-Wallis test; mean \pm SEM, Syn ${ }^{+} \mathrm{PSD}^{+} 5^{+}$puncta, $p=0.0284, \mathrm{UN}=1 \pm$ $0.11, \mathrm{MIA}=0.87 \pm 0.13, \mathrm{HI}=0.74 \pm 0.08, \mathrm{MIA} / \mathrm{HI}=0.34 \pm 0.1$; Syn $^{+}$puncta, $p=0.1427, \mathrm{UN}=1 \pm 0.11, \mathrm{MIA}=0.89 \pm 0.06, \mathrm{HI}=$ $0.74 \pm 0.02$, MIA/HI $=0.85 \pm 0.03$; $\mathrm{PSD}^{+} 5^{+}$puncta, $p=0.0 .0289$, $\mathrm{UN}=1 \pm 0.11, \mathrm{MIA}=0.7 \pm 0.11, \mathrm{HI}=0.89 \pm 0.04, \mathrm{MIA} / \mathrm{HI}=$ $0.53 \pm 0.08)$. Likewise, immunoblots showed significant reduction of PSD-95 and Homer-1, two ASD-related postsynaptic proteins (Tsai et al., 2012; Lelieveld et al., 2016), and the Rett syndrome protein $\mathrm{MeCP} 2$, but not synaptotagmin, in the ipsilateral hippocampus at $7 \mathrm{~d}$ after MIA/HI (Fig. $2 \mathrm{H}, \mathrm{I} ; n=3$ for each group; Kruskal-Wallis test; Syn protein levels, $p=0.3529$; PSD-95 protein levels, $p=0.048$; Homer 1 protein levels, $p=0.0409$; MeCP2 protein levels, $p=0.0462$ ). In contrast, MIA and pure-HI had no consistent effects on the expression of PSD-95, Homer-1, and MeCP2 (Fig. 2H,I). Further, the pattern of selective reduction of PSD-95 and Homer-1 in the MIA/HI-injured offspring hippocampus persisted into 2 months of age, when the animals showed impaired sociability and repetitive behaviors (Fig. 2J,K; $n=4$ males for each group; Kruskal-Wallis test; Syn protein levels, $p=0.7111$; PSD-95 protein levels, $p<0.0001$; Homer1 protein levels, $p<0.0001$ ). Of note, the presynaptic protein synaptotagmin is largely unaffected by MIA/HI, despite reduced PSD-95 and Homer-1 expression, 
A

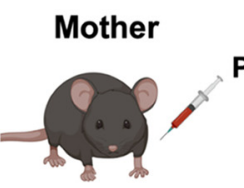

$$
\text { 1st hit (MIA) }
$$

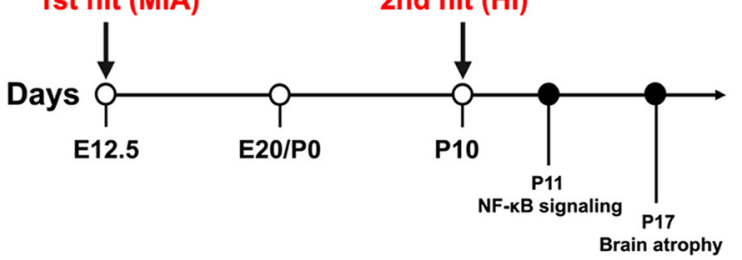

D

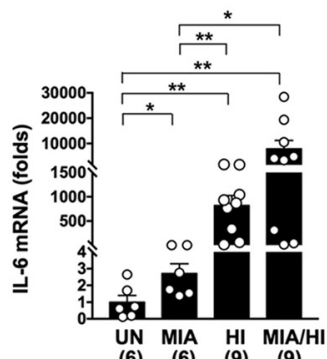

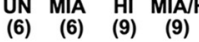

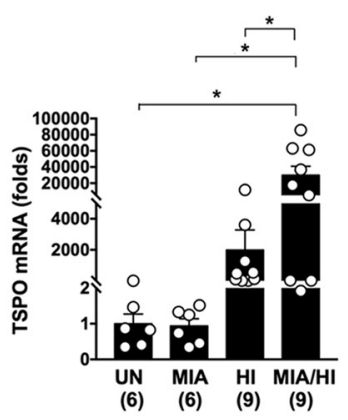

E

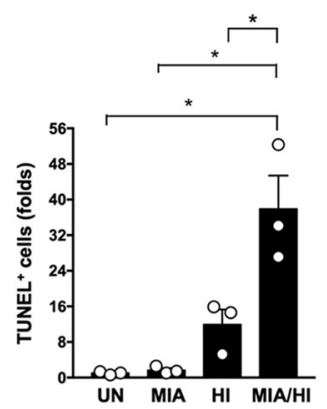

Brain atrophy

Offspring

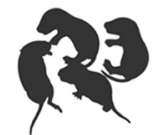

2nd hit (HI)
B

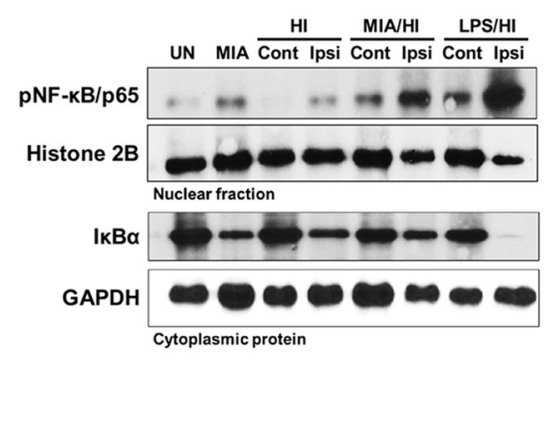

C

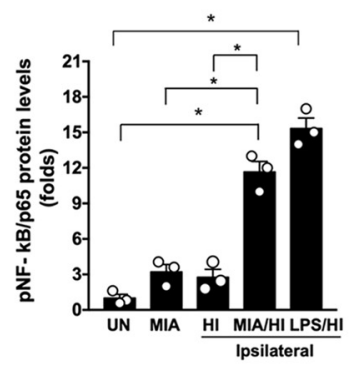

UN MIA HI MIA/H

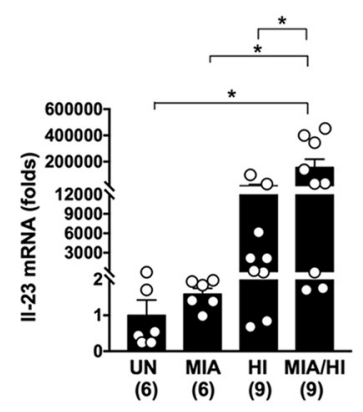

F

MIA HI MIA/HI

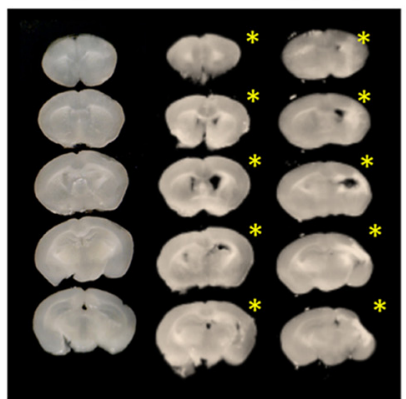

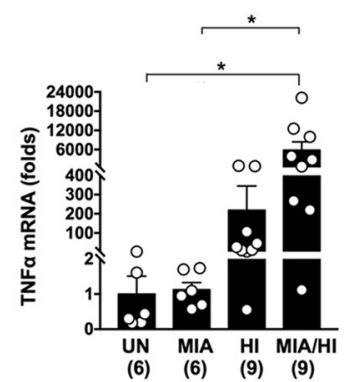

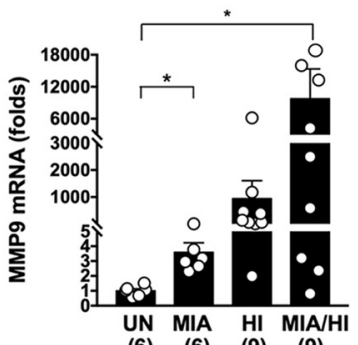

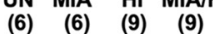
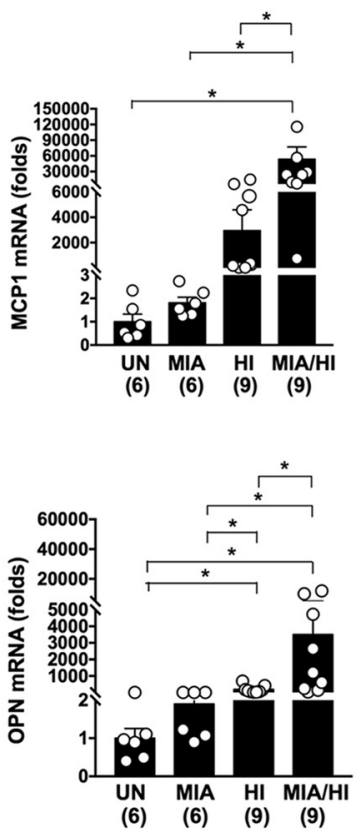

G

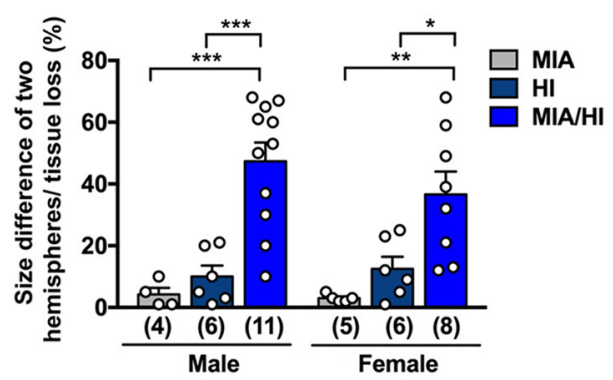

Figure 1. MIA and neonatal $H I$ have synergistic effects on NF $\kappa$ B signaling, proinflammatory cytokine production, and brain damage. $A$, Schematic diagram of the two-hit MIA/HI mouse model. $B, C$, MIA only slightly elevated the NF $\kappa$ B signaling activity in the uninjured P10 offspring but markedly amplified the responses to $\mathrm{HI}$ in ipsilateral hemisphere at $24 \mathrm{~h}$ recovery. Shown are the typical immunoblot against the nuclear phosphorylated NF $\kappa$ B and cytoplasmic $I_{\kappa} \mathrm{B} \alpha$ in each condition ( $n=3$ from three litters). $C$, Quantification and statistical analysis. Data are mean \pm SEM. $* p<0.05$ (Kruskal-Wallis test followed by Dunn's multiple comparison test). D, MIA elevated the IL-6, IL-17 $\alpha$, and MMP-9 transcripts in P10 offspring brains, whereas the dual MIA/HI injury caused a significant increase of the mRNA levels in ipsilateral hemisphere for IL-6, IL-17 $\alpha, M M P-9, T N F \alpha, I L-23, T S P O, M C P 1$, and OPN compared with pure-HI injury at $24 \mathrm{~h}$ recovery. UN, $n=6 ;$ MIA, $n=6 ; \mathrm{HI}, n=9 ; \mathrm{MIA} / \mathrm{HI}, n=9$. Data are mean \pm SEM. $* p<0.05 ; * * p<0.01$; one-way ANOVA and Tukey's multiple comparisons post hoc test. $\boldsymbol{E}$, The combined $\mathrm{MIA} / \mathrm{HI}$ insult induced more TUNEL-positive cell death in the ipsilateral cerebral cortex at $24 \mathrm{~h}$ recovery than MIA or pure-HI $(n=3)$. Data are mean \pm SEM. $* p<0.05$ (Kruskal-Wallis test followed by Dunn's multiple comparison test). $\mathbf{F}, \mathbf{G}$, Representative brain sections in the MIA offspring and post-HI or post-MIA/HI mice at $7 \mathrm{~d}$ of recovery. $* H I-$ or MIA/HI-injured hemisphere. Quantification of bilateral brain size difference in the MIA offspring (no tissue loss) or the percentage of brain atrophy (to the contralateral hemisphere) in $\mathrm{HI}$ - and MIA/HI-injured neonates of both sexes at $7 \mathrm{~d}$ of recovery (P17). There is no gender difference of tissue loss. Data are mean \pm SEM in both sexes. $* p<0.05 ; * * p<0.01 ; * * * p<0.0001$; two-way ANOVA followed by Tukey's multiple comparisons post hoc tests. 
A

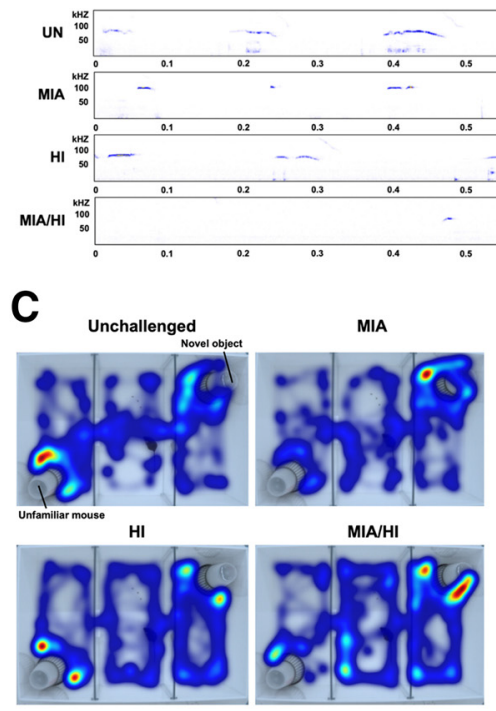

E

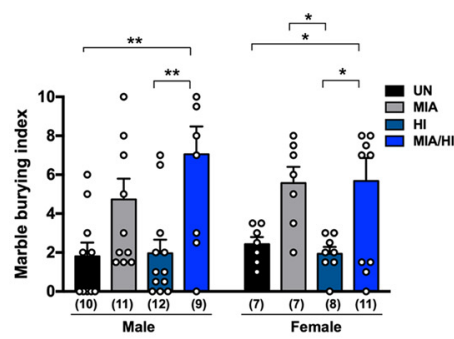

H

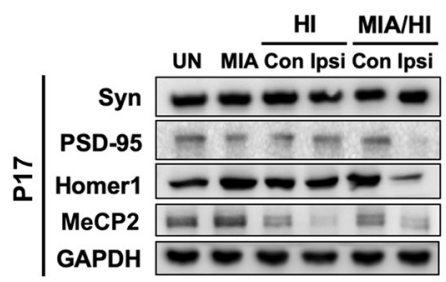

J

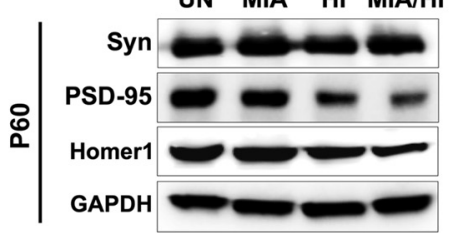

B

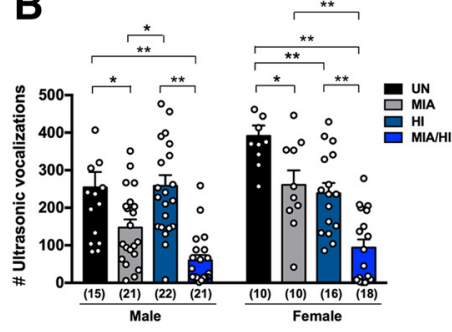

D

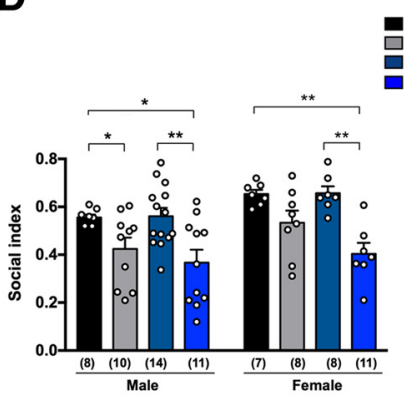

UIA

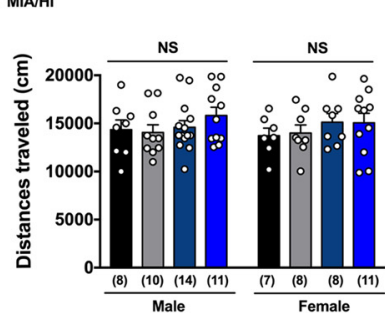

F

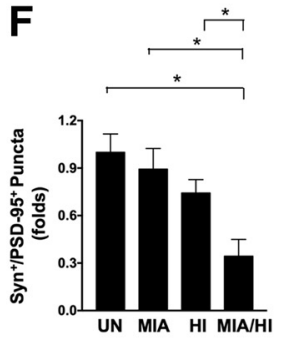

G

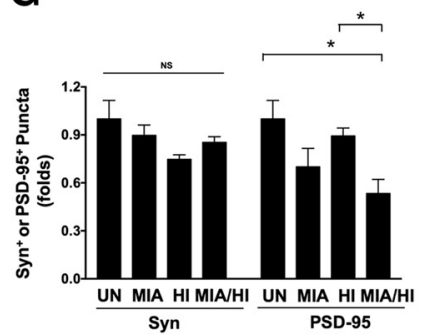

I
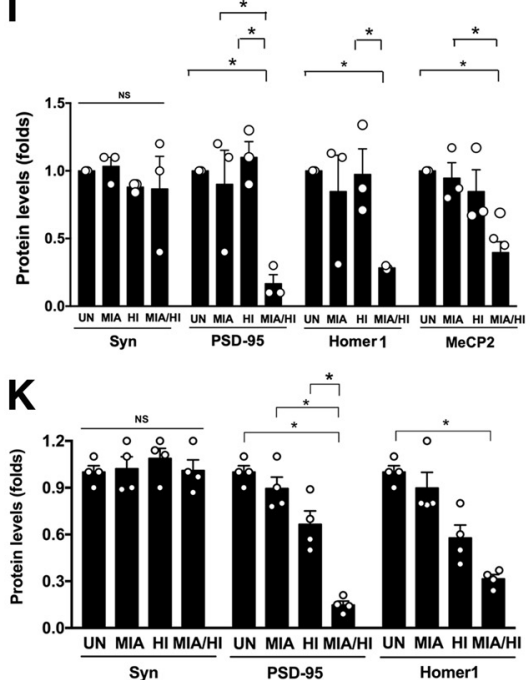

Figure 2. Dual-MIA/HI injury promotes autistic-like behaviors and synaptic defects. $\boldsymbol{A}$, Representative USV plots for P11 mice in the indicated treatment groups. Note severe reduction of USV in the MIA/HI-injured group. $\boldsymbol{B}$, The total numbers of USVs emitted by P11 mice during 3 min in each group were compared. Data are mean \pm SEM for both sexes. $* p<0.05 ; * * p<0.01$; two-way ANOVA followed by Tukey's multiple comparisons post hoc tests. The group sizes in males: UN (unchallenged, $n=15)$, MIA $(n=21)$, HI $(n=22)$, and MIA/HI ( $n=21)$; in females, UN $(n=10)$, MIA $(n=10), \mathrm{HI}(n=16)$, and MIA/HI $(n=18)$. C, $\boldsymbol{D}$, Comparison of social interactions and the total traveled distance in the three-chamber test. After 5 min acclimatization, 8 -weekold adult mice in each group were allowed to explore all three chambers for $10 \mathrm{~min}$. The $\mathrm{SI}$ was calculated as [time exploring novel mouse]/[time exploring novel mouse plus the inanimate object], as previously described (Pietropaolo et al., 2011). Data are mean \pm SEM for both sexes. $* p<0.05 ; * * p<0.01$; two-way ANOVA followed by Tukey's multiple comparisons post hoc tests. The sample sizes in males: UN $(n=8)$, MIA $(n=10)$, HI $(n=14)$, and MIA/HI $(n=11)$; in females, UN $(n=7)$, MIA $(n=8)$, HI $(n=8)$, and MIA/HI $(n=11)$. $\boldsymbol{E}$, The repetitive marble-burying behavior was measured in a 15 min testing session. The MIA offspring and post-MIA/HI mice showed increased marble-burying behaviors than UN mice, while HI-injured mice showed no such stereotyped behaviors. Data are mean \pm SEM for both sexes. $* p<0.05 ; * * p<0.01$; two-way ANOVA followed by Tukey's multiple comparisons post hoc tests. The group sizes in males: UN $(n=10)$, MIA $(n=11)$, HI $(n=12)$, and MIA/HI $(n=9)$; in females, UN $(n=7)$, MIA $(n=7)$, HI $(n=8)$, and MIA/HI $(n=11)$. $\boldsymbol{F}, \boldsymbol{G}$, Quantitation of anti-Synaptotagmin (Syn), antiPSD-95, and colocalized anti-Syn/anti-PSD-95 puncta in the hippocampal CA3 sector of P17 mice in each experimental group ( $n=3$ from three litters). Data are mean \pm SEM. $* p<0.05$ (Kruskal-Wallis test followed by Dunn's multiple comparison test). $\boldsymbol{H}, \mathbf{I}$, Immunoblotting analysis of presynaptic (Syn) and postsynaptic markers (PSD-95, Homer1), and the Rett syndrome protein MeCP2 in the hippocampus of uninjured, MIA offspring, post-HI, and post-MIA/HI mice at P17. Quantification showed that only dual-MIA/HI led to significant reduction of PSD-95, Homer1, and MeCP2 ( $n=3$ for each group). Data are mean \pm SEM. $* p<0.05$ (Kruskal-Wallis test followed by Dunn's multiple comparison test). $\boldsymbol{J}, \boldsymbol{K}$, Immunoblotting analysis of presynaptic (Syn) 
suggesting unique postsynaptic mechanisms that may inspire autistic-like behaviors.

\section{Dual MIA/HI boosts immune infiltrates and macrophages/ ameboid microglia in the brain}

Greater immune responses to MIA/HI may arise in part from increased immune infiltrates in the neonatal brains, similar to the responses to combined LPS/HI injury (Yang et al., 2013). To test this scenario, we used flow cytometry to compare immune infiltrates in the brain parenchyma of MIA offspring, post-HI, and post-MIA/HI neonates at P14 (i.e., $4 \mathrm{~d}$ after $\mathrm{HI}$ ). Figure $3 \mathrm{~A}$ shows the gating strategy and the representative cytometry plots of unchallenged (UN), MIAalone, and both hemispheres of $\mathrm{HI}$ - and MIA/HI-injured mouse brains. Figure $3 B, C$ shows the percentage and numbers of neutrophils $\left(\mathrm{CD} 45^{\mathrm{hi}} \mathrm{CD} 11 \mathrm{~b}^{+} \mathrm{Ly}_{6 \mathrm{G}}{ }^{+}\right)$, monocyte/macrophages $\left(\mathrm{CD} 45^{\mathrm{hi}} \mathrm{CD} 11 \mathrm{~b}^{+} \mathrm{Ly}_{6 \mathrm{G}}^{-}\right), \mathrm{CD}^{+} \mathrm{T}$ cells $\left(\mathrm{CD} 45^{+} \mathrm{CD} 11 \mathrm{~b}^{-}\right.$ $\left.\mathrm{CD}^{+} \gamma \delta \mathrm{TCR}^{-}\right)$, and $\gamma \delta \mathrm{T}$ cells $\left(\mathrm{CD} 45^{+} \mathrm{CD} 11 \mathrm{~b}^{-} \mathrm{CD}^{+} \gamma \delta \mathrm{TCR}^{+}\right)$. This analysis showed comparable percentages and cell counts of these immune infiltrates in the unchallenged, MIA offspring, and $\mathrm{HI}$-injured neonates (in both hemispheres). For MIA/HI-injured neonates, only the ipsilateral hemispheres contained significantly increased neutrophils, monocyte/macrophages, CD3 ${ }^{+}$ $\mathrm{T}$ cells, and $\gamma \delta \mathrm{T}$ cells (Fig. $3 B, C, \mathrm{UN}=6, \mathrm{MIA}=11, \mathrm{HI}=9$, $\mathrm{MIA} / \mathrm{HI}=7$, as indicated; one-way ANOVA; Fig. $3 B$, neutrophil percentage, $F_{(5,43)}=3.341, p=0.0123$; monocyte $/ \mathrm{macro-}$ phage percentage, $F_{(5,43)}=162.6, p<0.0001 ; \mathrm{CD}^{+} \mathrm{T}$ cells percentage, $F_{(3,29)}=9.656, p<0.0001 ; \gamma \delta \mathrm{T}$ cells percentage, $F_{(5,43)}=13.84, p<0.0001 ; 3 \mathrm{C}$, neutrophils, $F_{(5,43)}=4.219$, $p=0.0136 ;$ monocyte/macrophages, $F_{(3,29)}=13.6, p<0.0001$; $\mathrm{CD}^{+}$T cells, $F_{(3,29)}=14.81, p<0.0001 ; \gamma \delta$ T cells, $F_{(3,29)}=7.291$, $p=0.0009)$. In addition, the microglia $\left(\mathrm{CD} 45^{\mathrm{int}} / \mathrm{CD} 11 \mathrm{~b}^{+} / \mathrm{Ly}_{6 \mathrm{G}}{ }^{-}\right)$ and monocyte/macrophages $\left(\mathrm{CD} 45^{\mathrm{hi}} / \mathrm{CD} 11 \mathrm{~b}^{+} / \mathrm{Ly}_{6 \mathrm{G}}{ }^{-}\right)$in postMIA/HI brains showed increased expression of Trem2 (Fig. 3D,E; $n=6-11$ in each group, as indicated; one-way ANOVA; monocyte/macrophages, $F_{(3,29)}=6.567, p=0.0016$; microglia; $F_{(3,29)}=$ $13.1, p<0.0001$ ), a microglial effector that is implicated in synapse elimination (Filipello et al., 2018).

We also used CX3CR $1^{G F P /+}$ mice to visualize the morphology of microglia in these conditions (Jung et al., 2000). Interestingly, ameboid microglial cells (AMCs) were detected in a rising incidence in the hippocampus of unchallenged (0/4), MIA offspring (2/11), post-HI (6/10), and post-MIA/HI (9/9) neonates at P17 (Fig. 3F). These AMCs formed clusters most frequently in the CA3 sector and expressed CD68 plus complement component 3 (Fig. $3 G$ ), suggesting proinflammatory and synapse-eliminating actions (Schafer et al., 2012; Wu et al., 2015). Together, these results suggest that combined $\mathrm{MIA} / \mathrm{HI}$ has a greater potency to provoke immune infiltrates and AMCs that may endanger the synaptic network in offspring brains.

\section{Dual MIA/HI potently attracts Otx2-expressing monocytes and elevates the PNN intensity}

To test the origins of the CX3CR $1^{G F P /+}$ AMC clusters, we used $C C R 2^{R P P /+} ; C X 3 C R 1^{G F P /+}$ mice (hereafter referred to as $\mathrm{R} / \mathrm{G}$

\footnotetext{
and postsynaptic markers (PSD-95, Homer1) in unchallenged, MIA offspring, post-HI, and post-MIA/HI mouse hippocampus in P60 mice. Quantification showed that only dual-MIA/HI insult led to significant reduction of PSD-95, Homer1 ( $n=4$ males for each group). Data are mean \pm SEM. $* p<0.05$ (Kruskal-Wallis test followed by Dunn's multiple comparison test). NS: not significant.
}

mice) to compare the influx of CCR $2^{\mathrm{RFP}+}$ monocytes in MIA-, HI-, and MIA/HI-injured neonatal brains (Saederup et al., 2010). This analysis showed near-absence of CCR2 ${ }^{\mathrm{RFP}+}$ monocytes in naive or MIA offspring brains, but many CCR2 $2^{\mathrm{RFP}+}$ cells coinciding with $\mathrm{CX} 3 \mathrm{CR} 1^{\mathrm{GFP}+} \mathrm{AMC}$ clusters in the ipsilateral hippocampus of post-MIA/HI, and to a lesser extent in HI-injured brains, at $7 \mathrm{~d}$ of recovery (Fig. $4 A, B ; n>6$ each for imaging and $n=3$ each for quantification; Kruskal-Wallis test, $p=0.0006$; $\mathrm{RFP}^{+}$cells, mean $\pm \mathrm{SEM}, \mathrm{UN}=0 \pm 0, \mathrm{MIA}=0.33 \pm 0.33$, $\mathrm{HI}=52.33 \pm 5.04, \mathrm{MIA} / \mathrm{HI}=108 \pm 25.14)$. Importantly, many CCR2 ${ }^{\mathrm{RFP}+}$ cells were also CX3CR1 ${ }^{\mathrm{GFP}+}$ (Fig. $4 A$, bottom row), raising the possibility of "monocyte-to-microglia transition" in the post-MIA/HI brains.

We also assessed the possibility of monocytic expression of Otx2, a homeoprotein that is usually synthesized only in the choroid plexus, but secreted globally to promote PNN formation (Spatazza et al., 2013), among these experimental groups. Interestingly, double-labeling showed increased anti-Oxt 2 immunosignals often in $\mathrm{CCR} 2^{\mathrm{RFP} /+}$ cells at $7 \mathrm{~d}$ after MIA/HI (Fig. $4 C, D ; n=3$ for each condition; Kruskal-Wallis test, $p=0.0002$; $\mathrm{RFP}^{+} \mathrm{Otx} 2^{+}$cells, mean \pm $\mathrm{SEM}, \mathrm{UN}=0.33 \pm 0.33, \mathrm{MIA}=0.66 \pm 0.66, \mathrm{HI}=8.33 \pm 0.88, \mathrm{MIA} /$ $\mathrm{HI}=82.66 \pm 6.83$ ). Moreover, the MIA/HI-injured brains contained the highest number of WFA-wrapped hippocampal PV neurons at $7 \mathrm{~d}$ of recovery (Fig. $4 E, F ; n=3$ each for quantification; Kruskal-Wallis test, $p=0.0002 ; \mathrm{WFA}^{+} \mathrm{PV}^{+}$cells, mean $\pm \mathrm{SEM}$, $\mathrm{UN}=1.66 \pm 1.66, \mathrm{MIA}=13.3 \pm 1.66, \mathrm{HI}=26.66 \pm 6, \mathrm{MIA} / \mathrm{HI}=$ $61.66 \pm 8.81)$. These results suggest that invading monocytes after MIA/HI may constitute an ectopic source of Otx2 to promote PNN formation in neonatal brains.

\section{Infiltrating monocytes undergo in situ reprograming of transcription and metamorphosis}

The prevalence of CX3CR1 ${ }^{\mathrm{GRP}+} \mathrm{CCR} 2^{\mathrm{RFP}+}$ cells in post-MIA/ $\mathrm{HI}$ brains raised the possibility of "monocyte-to-microglia transition," as recently shown in neonatal stroke (Chen et al., 2020). To test this scenario, we crossed CCR2-CreER(T2) transgenic mice with ROSA26R-CAG-ZsGreen (Ai6) reporter mice, and dosed the bitransgenic MIA offspring with tamoxifen $(10 \mathrm{mg} / \mathrm{kg} /$ day) at P8-P9 before HI insult at P10, to identify $\mathrm{GFP}^{+}$monocytes and their derivatives in post-MIA/HI brains. The CCR2-CreER-based fate-mapping method has the advantage of permanently labeling monocyte derivatives, which may "disappear" in CCR $2^{\mathrm{RFP} /+}$ mouse brains if/when they no longer express the monocytic marker CCR2 (Saederup et al., 2010).

In CCR2-CreER ${ }^{\text {tg/+ }} ; \mathrm{Ai}^{\mathrm{tg} /+}$ mice (referred to as bitransgenic CCR2-CreER mice) of MIA offspring, tamoxifen dosing produced $\mathrm{GFP}^{+}$monocytes only in the choroid plexus (Fig. 5A). In contrast, tamoxifen-dosed and MIA/HI-injured bitransgenic CCR2-CreER mice contained copious $\mathrm{GFP}^{+}$cells in the ipsilateral cerebral cortex and hippocampus, but not in the contralateral hemisphere, at 4 or $30 \mathrm{~d}$ of recovery (Fig. $5 B-E, L-O ; n>4$ for each time point). The $\mathrm{GFP}^{+}$monocyte derivatives mostly displayed an ameboid cell shape and were labeled by anti-Iba1, antiCD68, and anti-TNF $\alpha$ at $4 \mathrm{~d}$ after MIA/HI (Fig. $5 F-K$, arrows), suggesting proinflammatory actions at this age. By $30 \mathrm{~d}$ after MIA/HI, many $\mathrm{EGFP}^{+}$monocyte derivatives coalesced to form large clusters in the ipsilateral cerebral cortex, but many remained individually visible and adopted a ramified microglialike morphology (Fig. $5 L-U$ ). Yet, a subset of non-AMC monocyte derivatives still expressed the proinflammatory markers CD68 and TNF $\alpha$ at $30 \mathrm{~d}$ after MIA/HI (Fig. 5R-U). Further, both ameboid and ramified monocyte derivatives expressed the 


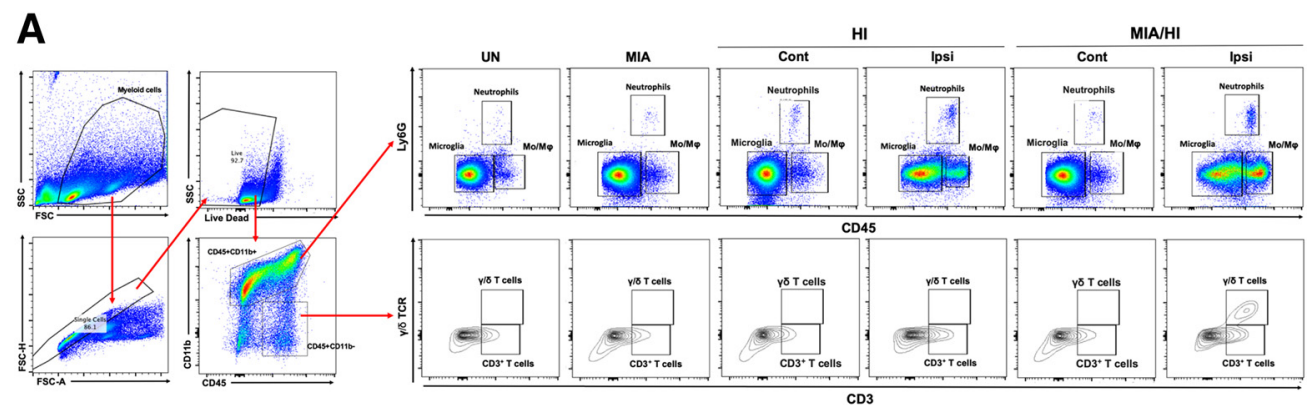

B

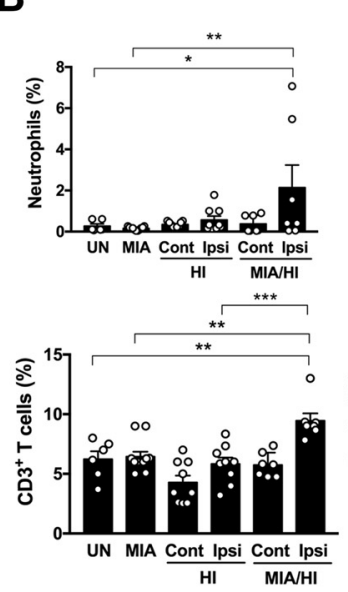

D

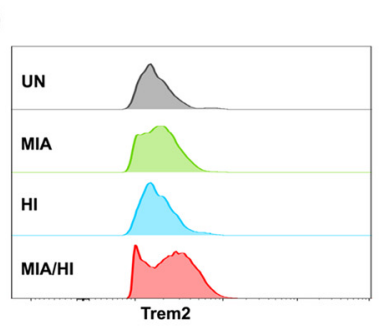

G
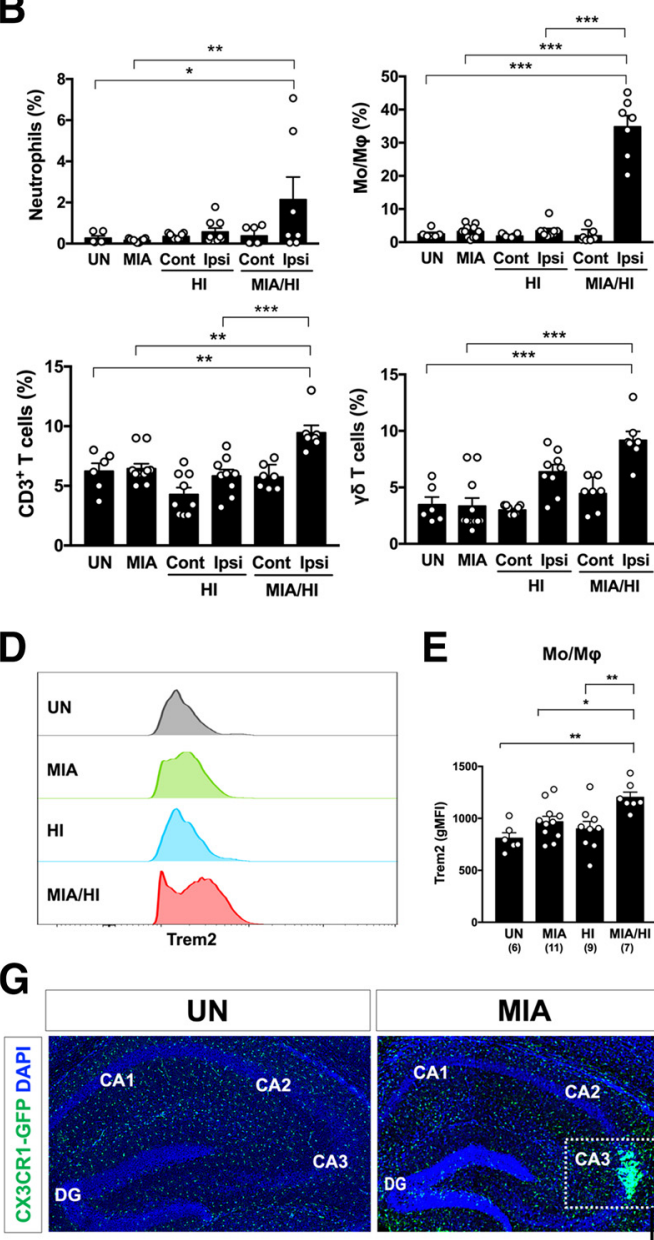

C

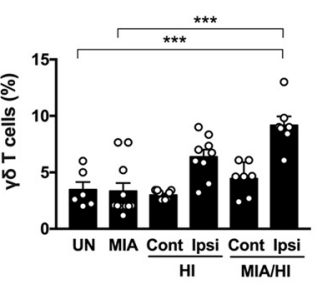

E
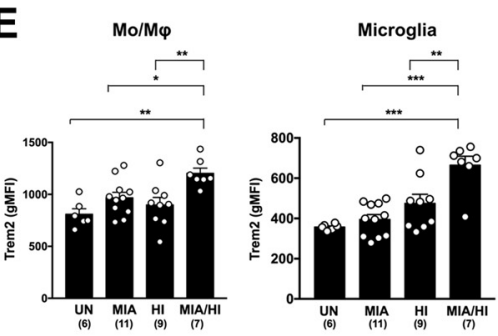

$\mathbf{F}$
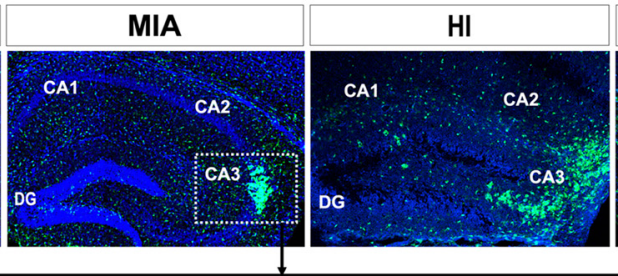
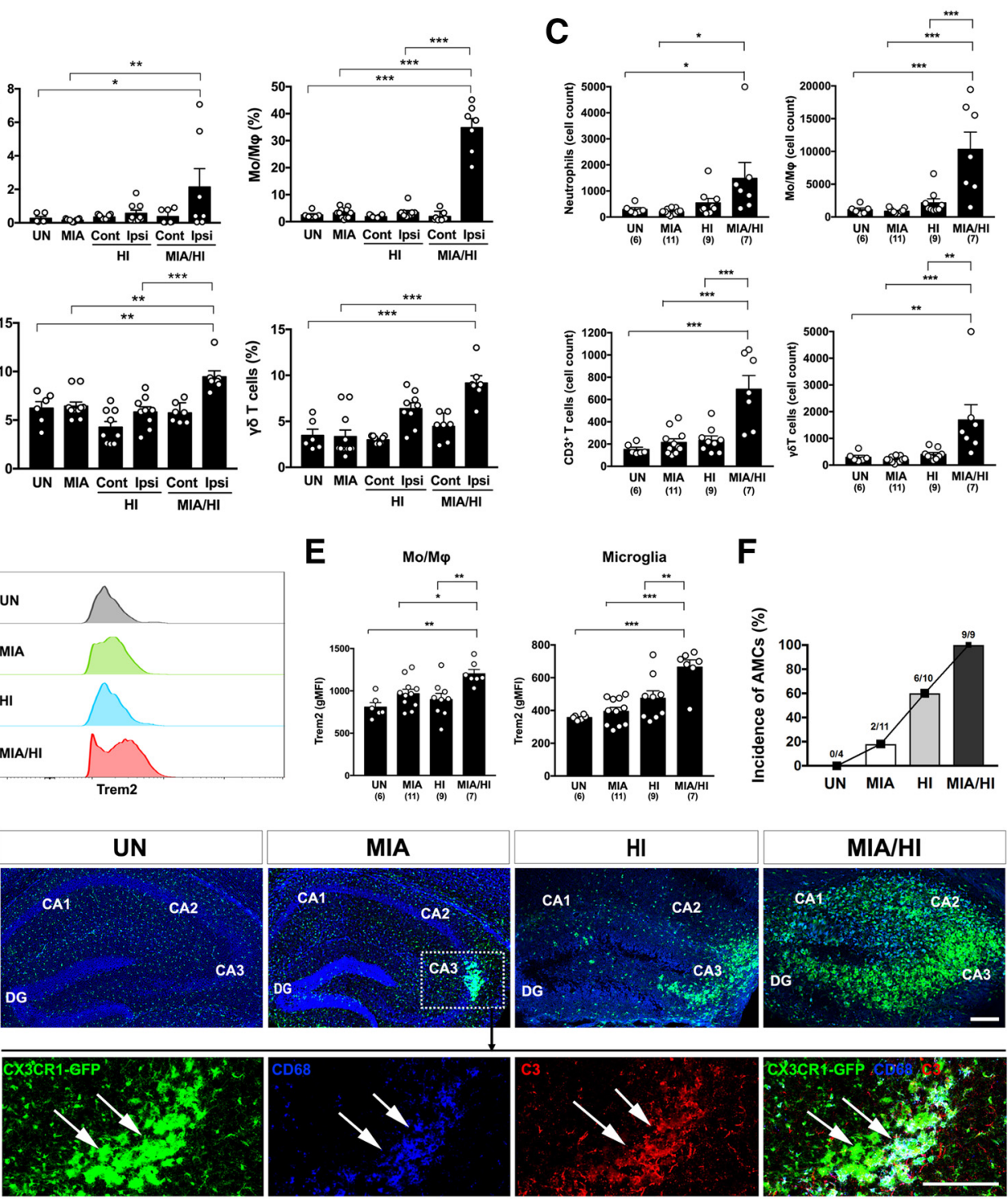

Figure 3. Dual-MIA/HI increases immune infiltrates, particularly monocytes, into the brain. $A$, The gating strategy and representative flow cytometry plots of immune infiltrates in brain pa-

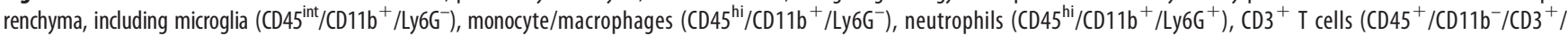

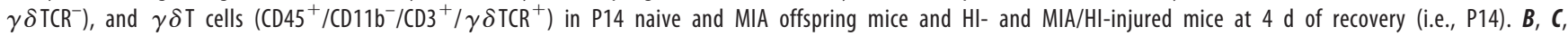
Quantification of the immune infiltrates in brain parenchyma in P14 naive and MIA offspring plus HI- and MIA/HI-injured mice at $4 \mathrm{~d}$ of recovery (i.e., P14). Note the significant increase of monocyte/macrophages $(\mathrm{Mo} / \mathrm{M} \varphi), \mathrm{CD}^{+} \mathrm{T}$, and $\gamma \delta \mathrm{T}$ cells in brain parenchyma after dual-MIA/HI compared with pure-HI injury. $N=6-11$ for each group, as indicated. Data are mean \pm SEM. $* p<0.05 ; * * p<0.01 ; * * * p<0.0001$; one-way ANOVA followed by Tukey's multiple comparisons post hoc tests. $\boldsymbol{D}, \boldsymbol{E}$, Histograms of the antiTrem2-labeling intensity in $\mathrm{Mo} / \mathrm{M} \varphi$ and microglia in each condition ( $n=6-11$ per group, as indicated). Note greater anti-Trem2 labeling in both $\mathrm{Mo} / \mathrm{M} \varphi$ and microglia in postMIA/HI than post-HI neonate brains. Data are mean \pm SEM. $* p<0.05 ; * * p<0.01 ; * * * p<0.0001$; one-way ANOVA followed by Tukey's multiple comparisons post hoc tests. $\boldsymbol{F}$, Quantitative comparison of the incidence of hippocampal ameboid microglial clusters (AMCs) in UN $(n=4)$, MIA $(n=11)$, pure-HI $(n=10)$, and MIA/HI-injured neonate brains $(n=9)$. G, Representative images of the absence (in UN) and presence of hippocampal CX3CR1 ${ }^{\text {GPP }+}$ AMCs in each group. Bottom, AMCs were labeled by anti-CD68 and anti-complement component 3 (C3) ( $n=3$ for each group). Scale bar, $100 \mu \mathrm{m}$. 
A

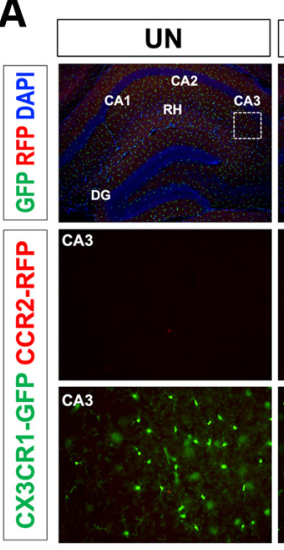

C

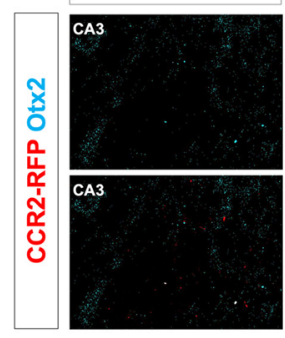

E

땔
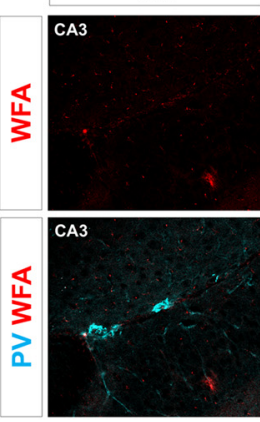

MIA

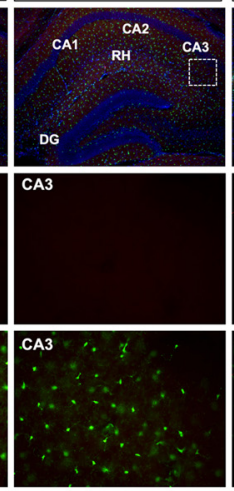

MIA

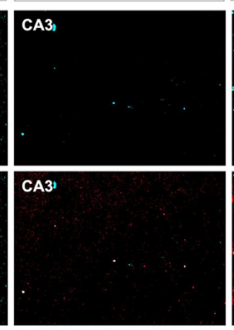

MIA

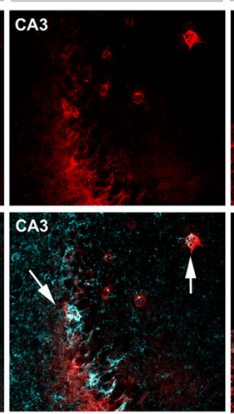

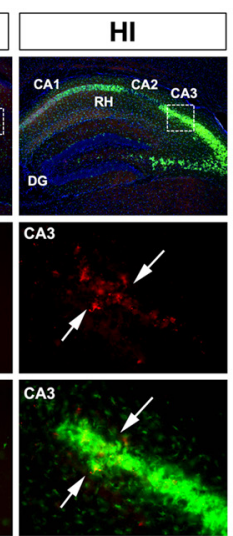

HI

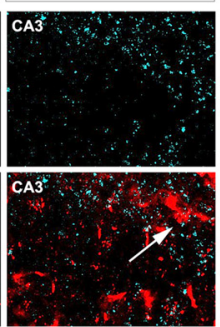

HI

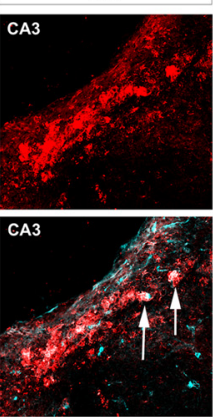

MIA/HI

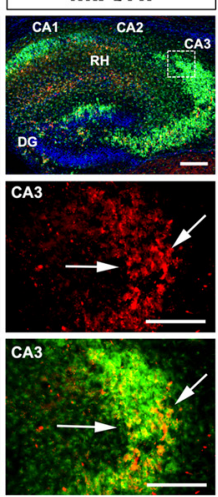

MIA/HI

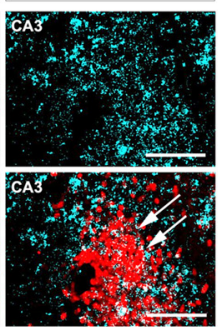

B

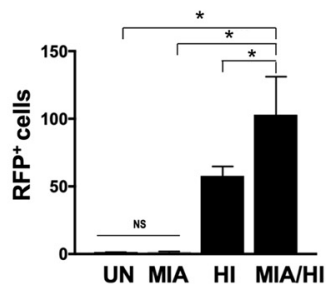

D

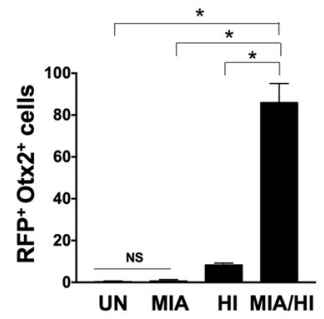

$\mathbf{F}$

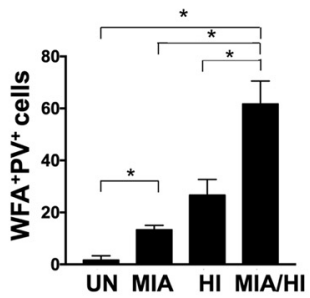

Figure 4. Dual-MIA/HI boosts monocytic infiltrates, Otx2 expression, and PNNs in the brain. $\boldsymbol{A}-\boldsymbol{D}, C\left(R 2^{R F P /+} ; C X 3 C R 1^{G F P /+}(\mathrm{R} / \mathrm{G})\right.$ mice were used to compare monocyte infiltration $(\boldsymbol{A}, \boldsymbol{B})$ and 0tx2 expression $(\boldsymbol{C}, \boldsymbol{D})$ under the indicated conditions in P17 hippocampus (i.e., $7 \mathrm{~d}$ after the $\mathrm{HI}$ insult). $\boldsymbol{A}$, Rectangles in the $\mathrm{CA} 3$ area in the top row were magnified in the two bottom rows and for quantification. Note the increase of $\mathrm{RFP}^{+}$monocytic infiltrates often colocalized as GFP ${ }^{+}$AMCs (second and third row in $\boldsymbol{A}$ ) after combined MIA/HI ( $\boldsymbol{B}, n=3$ from three litters for each group). The post-MIA/HI neonates also showed the largest number of $0 \mathrm{tx} 2^{+}$and $0 \mathrm{tx} 2^{+} \mathrm{RFP}^{+}$cells $(\boldsymbol{C}, \boldsymbol{D} ; n=3$ from three litters for each group). $\boldsymbol{E}, \boldsymbol{F}$, Immunostaining of WFA, and PV in the hippocampus and CA3 sector of P17 mice in indicated conditions. Post-MIA/HI neonate brains contained the largest number of WFA-enwrapped PV ${ }^{+}$interneurons $(n=3$ for each). Data are mean \pm SEM. $* p<0.05$ (Kruskal-Wallis test and Dunn's multiple comparison test). Scale bar, $100 \mu \mathrm{m}$. NS: not significant.

Sall1 mRNAs, a presumed "microglial signature gene," at 30 $\mathrm{d}$ after MIA/HI (Fig. 6A; $n=3$ ) (Bennett et al., 2016; Prinz et al., 2019).

We also derived bitransgenic heterozygous CCR2-CreERTRAP mice by crossing CCR2-CreER(T2) mice with the ROSA26R-EGFP/RpL10A mice. The CCR2-CreER-TRAP mice after tamoxifen-dosing enabled us to use TRAP to isolate mRNAs in monocytes and derivatives from 4 to $14 \mathrm{~d}$ after MIA/ HI for transcriptome analysis (Heiman et al., 2014). Compared with CCR ${ }^{+}$monocytes in the bone marrow and peripheral blood, the invading monocytes showed a rapid decline of CCR2 (a monocyte marker) and a surge, then decline, of TNF $\alpha$ mRNAs, plus an inverse increase of Sall1, Tmem119, and P2RY12 (all microglia marker genes) and Otx2 (implicated in PNN formation) from $4 \mathrm{~d}$ to $14 \mathrm{~d}$ after MIA/HI, while the Otx1 mRNAs (unrelated to PNNs) showing random fluctuations (Fig. $6 B ; n=3$ for each condition; each sample was pooled from three mouse brains; Kruskal-Wallis test; CCR2 mRNAs, $p=0.048$; Sall1 mRNAs, $p<0.0001$; Tmem119 mRNAs, $p=0.047$; TNF $\alpha$ mRNAs, $p=0.0003$; P2RY12 mRNAs, $p=0.0208$; Otx 2 mRNAs, $p<0.0001$; Otx 1 mRNAs, $p=0.9103)$. Congruently, immunofluorescence labeling showed more robust and discrete Tmem119 and P2RY12 expression in $\mathrm{GFP}^{+}$monocyte derivatives at $30 \mathrm{~d}$ than $4 \mathrm{~d}$ after MIA/HI (Fig. 6C,D). Fate-mapping using bitransgenic CCR2-CreER mice also confirmed the expression of Otx 2 in both amoeboid and ramified monocyte derivatives in $30 \mathrm{~d}$ post-MIA/HI brains (Fig. $7 A ; n=3$; Student's $t$ test, $p=0.023$; Otx $2^{+}$cells, mean $\pm \mathrm{SEM}$, $\mathrm{UN}=4 \pm 1.52, \mathrm{MIA}=159 \pm 43.48)$. Further, the WFA-wrapped $\mathrm{PV}$ neurons were often surrounded by $\mathrm{GFP}^{+}$monocyte derivatives in the hippocampal CA3 sector at $30 \mathrm{~d}$ after MIA/ HI (Fig. $7 B ; n=3$ ). Together, these results support "monocyte-to-microglia transition" and monocytic Otx2 expression after MIA/HI injury. 

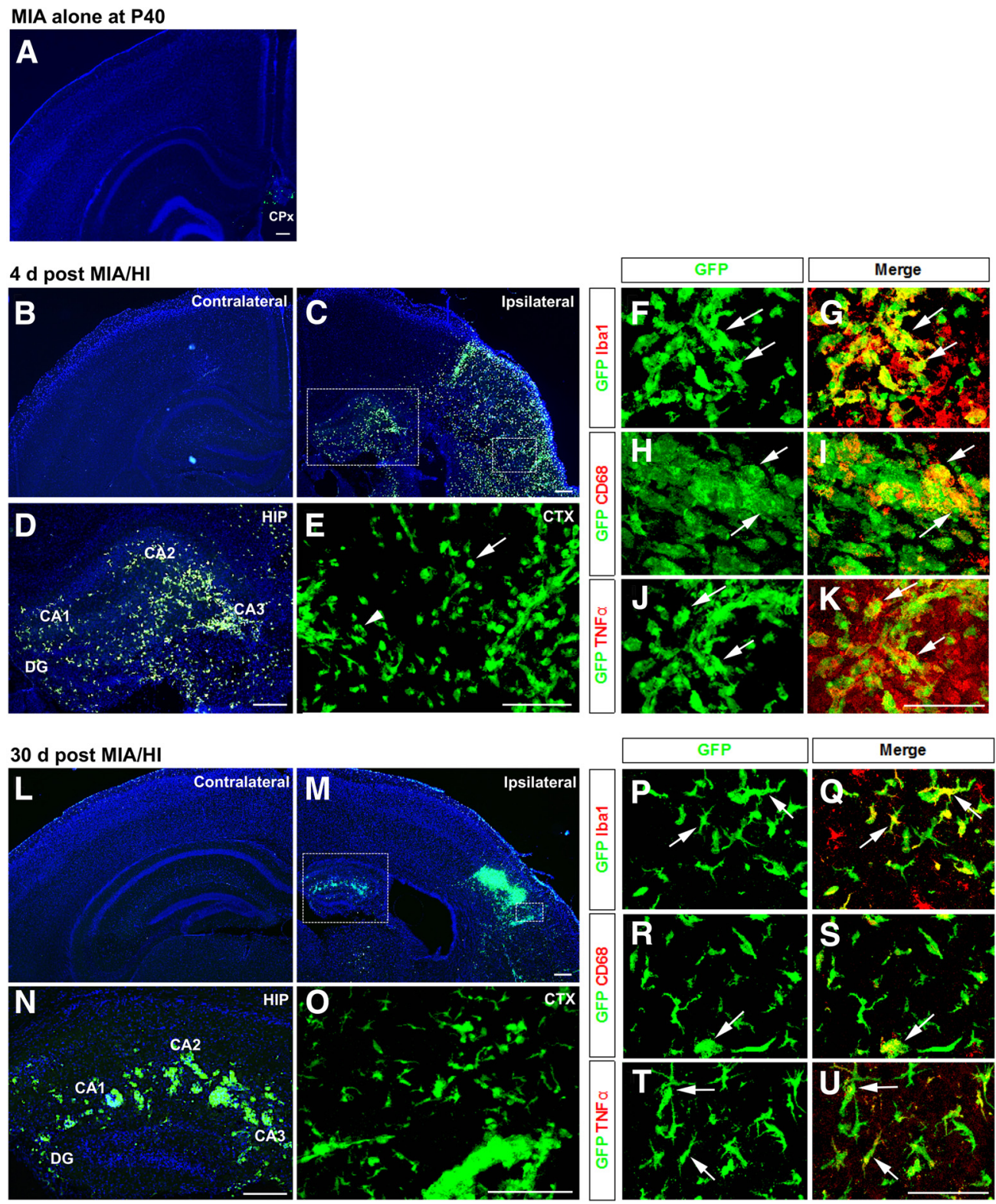

Figure 5. Fate-mapping experiments using CCR2-CreER(T2) mice showed morphologic alterations in post-MIA/HI brains. A, Infiltrating GFP ${ }^{+}$monocyte derivatives were present only the choroid plexus (CPx) of P40 MIA offspring brains, when tamoxifen was injected at P8 and P9. B-U, In contrast, tamoxifen-dosed and MIA/HI-injured bitransgenic CCR2-CreER mice contained copious $\mathrm{GFP}^{+}$cells in the ipsilateral cerebral cortex and hippocampus $(\boldsymbol{B}-\boldsymbol{E}, \boldsymbol{L}-\mathbf{O})$. At $4 \mathrm{~d}$ after MIA/HI, GFP ${ }^{+}$monocyte derivatives were populated in the ipsilateral, but not contralateral, hemisphere. The indicated rectangles are magnified as the hippocampal $\left(\boldsymbol{D}\right.$, HIP) and cerebral cortex $(\boldsymbol{E}, \mathbf{C T X})$ regions. The majority of GFP ${ }^{+}$monocyte derivatives showed a round shape $(\boldsymbol{E}-\boldsymbol{K}$, arrows) at $4 \mathrm{~d}$ after MIA/HI, and were labeled by anti-lba1 $(\boldsymbol{F}, \mathbf{G})$, anti-CD68 $(\boldsymbol{H}, \boldsymbol{I})$, or anti-TNF $\alpha(J, K)$. At $30 \mathrm{~d}$ of recovery, many monocyte derivatives adopted a ramified microglia-like morphology but remained colabeled by anti-lba1, anti-CD68, or anti-TNF $\alpha$ (P-U, arrows). $n=3$ each for 4 and $30 \mathrm{~d}$ after MIA/HI. Scale bar, $100 \mu \mathrm{m}$.

Blocking monocytes infiltration attenuates MIA/HI-induced autistic-like behaviors

To test whether monocytic infiltrates promote PNNs and autisticlike behaviors after MIA/HI, we first compared the numbers of WFA-enwrapped PV neurons in the hippocampal CA3 sector between WT and $C C R 2^{R F P / R F P}$ mice. In homozygous $C C R 2^{R F P / R F P}$ mice, the CCR2 receptor is deleted, thus disabling monocytes to respond to the chemoattractant MCP1/CCL2 after injury (Saederup et al., 2010). This experiment showed that $C C R 2^{R F P / R F P}$ mice contained significantly fewer WFA-enwrapped hippocampal PV neurons than WT mice at $30 \mathrm{~d}$ after MIA/HI (Fig. $7 C ; n=4$ for each group; Kruskal-Wallis test, $p=0.0036$; mean $\pm \mathrm{SEM} \mathrm{WFA}^{+} \mathrm{PV}^{+}$ cells, $\mathrm{UN}=4 \pm 1$, WT-MIA/HI $=54 \pm 5.03, C C R 2^{R F P / R F P}$-MIA/ $\mathrm{HI}=10 \pm 1.45)$. Similarly, CCR2 ${ }^{\mathrm{RFP} / \mathrm{RFP}}$ mice of both sexes showed recovered USV at P11 (compare the results in Fig. $7 D$ to unchallenged mice in Fig. 2B), fewer repetitive behaviors plus improved social interactions than WT mice at P60 (Fig. $7 D-F$; $n=7-12$ per group; two-way ANOVA, USV, $F_{(2,46)}=$ $13.21, p<0.0001$; repetitive behaviors, $F_{(2,30)}=10.85, p=$ 0.0003 ; sociability, $\left.F_{(2,30)}=11.81, p=0.0002\right)$. Control experiments showed that unchallenged WT, CCR2 $2^{R F P /+}$, and $C C R 2^{R F P / R F P}$ mice showed no difference in USV, sociability, and repetitive behaviors at 2 months of age (Fig. $7 G-I$ ).

In addition, post-HI injection of $20 \mathrm{mg} / \mathrm{kg} \mathrm{RS} 102895$, a CCR2 antagonist (Mirzadegan et al., 2000), preserved USV at P11 and prevented repetitive behaviors plus aberrant sociability in both male and female post-MIA/HI mice at 2 months of age (Fig. $7 D-F ; n=5-9$, as indicated). These results suggest that CCR2 ${ }^{+}$ 
A
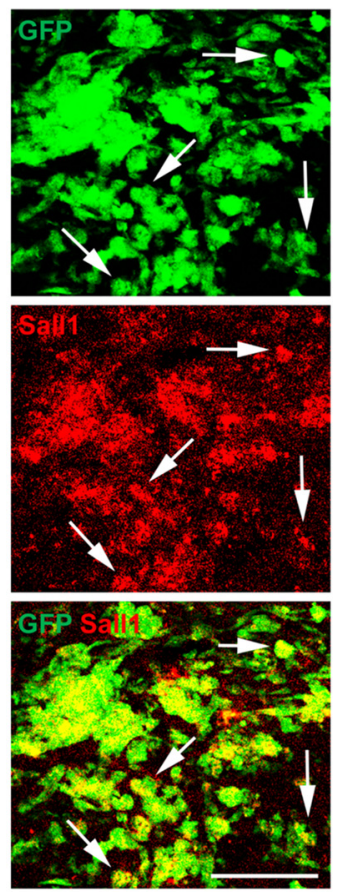

B
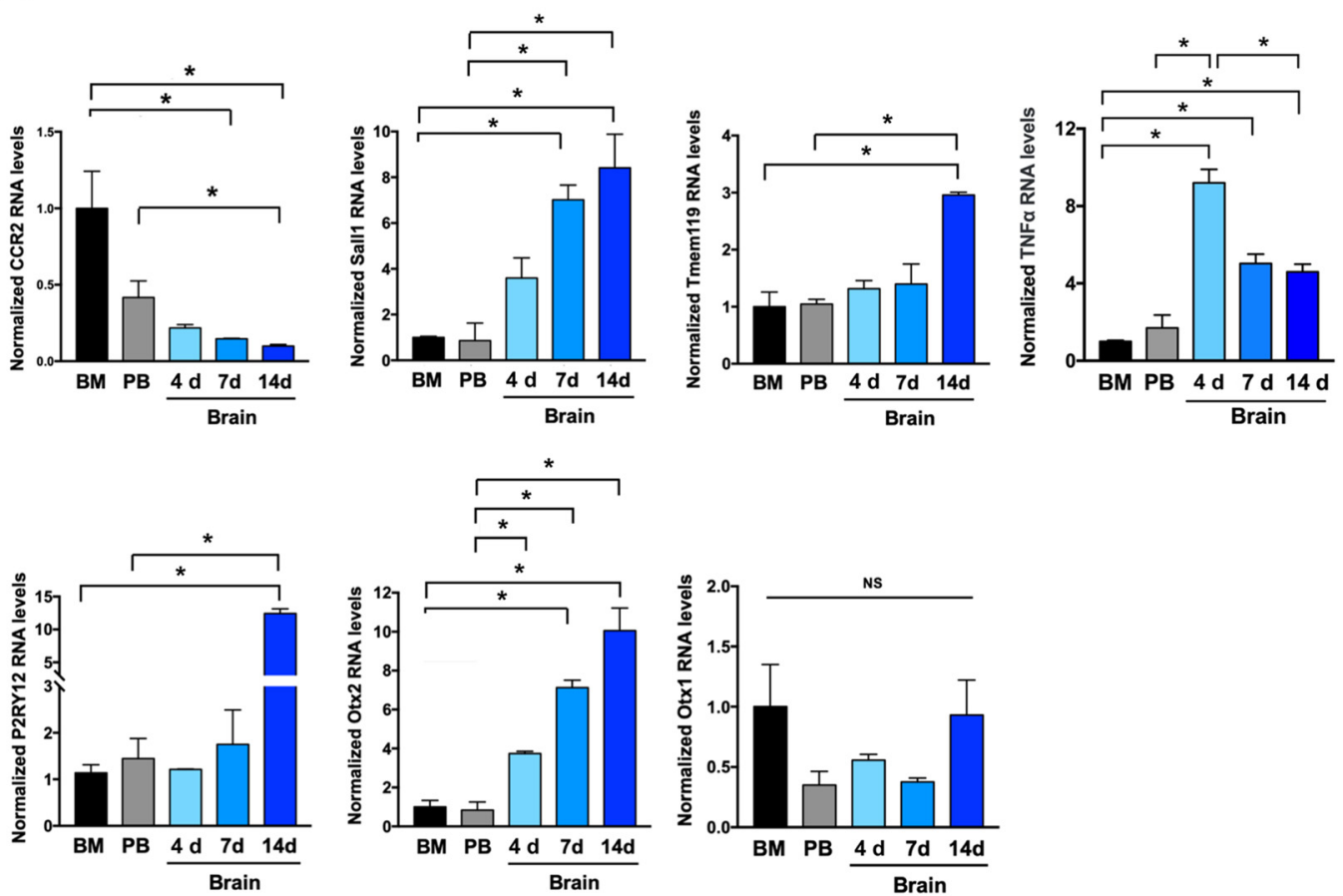
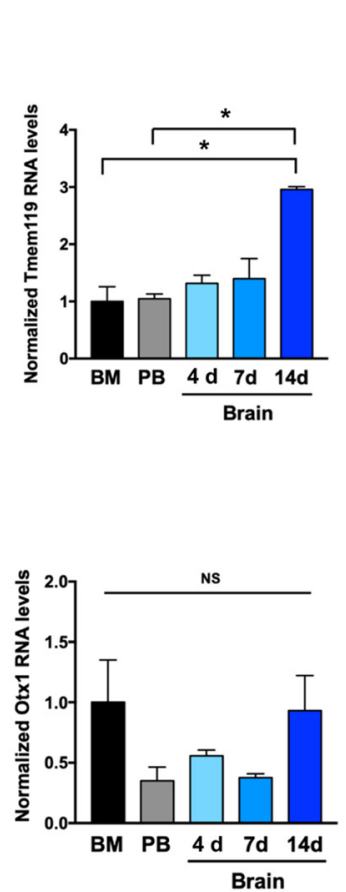

C

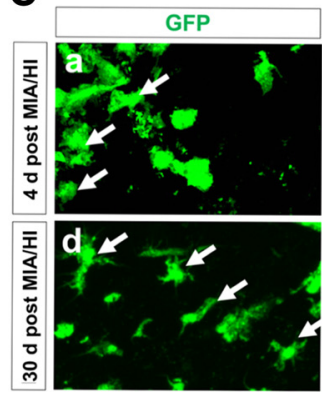

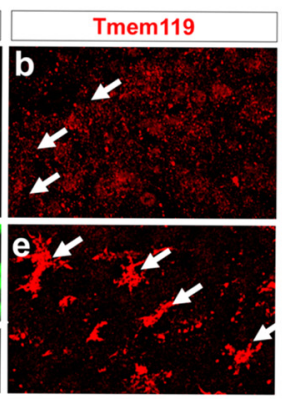

GFP Tmem119
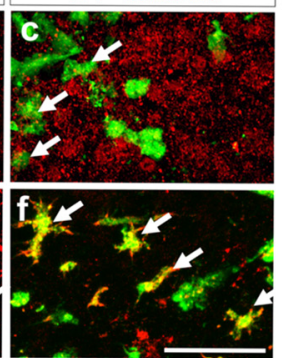

D

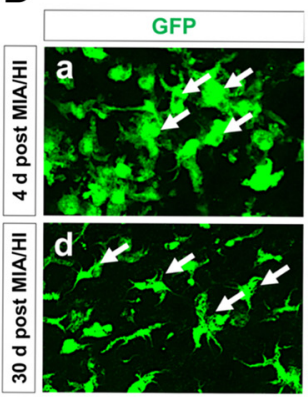

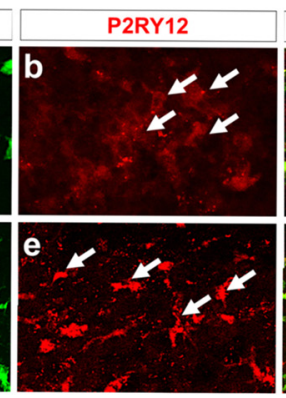

Figure 6. Monocyte derivatives gradually express "microglia-specific" markers and 0tx2 in post-MIA/HI brains. A, RNAscope ISH detected Sall1 mRNAs in GFP ${ }^{+}$monocyte derivatives in $30 \mathrm{~d}$ post-MIA/HI brains $(n=3)$. Scale bar, $100 \mu \mathrm{m}$. B, TRAP-based qRT-PCR analysis with CCR2-CreER(T2); ROSA26R-EGFP/RpL10A mice showed rapid decline of CCR2 mRNAs, a surge-then-decline of TNF $\alpha$ mRNA, and a gradual increase of Sall1, P2RY12, Tmem119, and Otx2 mRNAs in monocyte derivatives from 4-7 to $14 \mathrm{~d}$ post-MIA/HI brains compared with the expression levels in monocytes in the bone marrow (BM) and peripheral blood (PB). In contrast, monocyte derivatives showed no clear pattern of Otx1 mRNA alterations in post-MIA/HI brains. Data are mean \pm SEM for each condition $(n=3) . * p<0.05$ (Kruskal-Willis test followed by Dunn's multiple comparison test). C, D, Immunofluorescence labeling of Tmem119 and P2RY12, two microglial markers, became more robust in $\mathrm{GFP}^{+}$monocyte derivatives along with morphologic transition into a ramified cell shape from 4 to $30 \mathrm{~d}$ after MIA/HI. Shown are the representative images from $n=3$ for both post-MIA/HI dates. Scale bar, $100 \mu \mathrm{m}$. NS: not significant.

monocytes contribute to ectopic PNNs and ASD-like behaviors after MIA/HI injury.

\section{Discussion}

ASD is a heterogeneous group of neurodevelopmental disorders across multiple contexts that is presumably caused by complex interactions among multiple genetic, immune, and environmental factors (American Psychiatric Association, 2013). Accordingly, no animal model based on a single risk factor can capture all the cellular and behavioral features of ASD (Servadio et al., 2015). Yet, by focusing on the animal versions of core ASD symptoms (Malkova et al., 2012), one can test the interaction of relevant genetic, immune, and environmental factors to bridge the differences between experimental models and clinical symptoms. Using a novel two-hit model, the results of this study suggest cooperation between prenatal MIA and neonatal brain injury to promote autistic deficits that are contributed by monocytic infiltrates.
MIA-neonatal brain injury interactions in the pathogenesis of ASD

The idea that prenatal neuroimmune mechanisms may contribute to the onset of ASD originated from the finding of a higher prevalence of autism in children with congenital Rubella syndrome in the 1970s (Meyer, 2014; Estes and McAllister, 2016). This concept was further supported by the rodent MIA model, in which injection of virus-mimicking poly(I:C) at mid-gestation to dam leads to autistic-like behaviors in the offspring (Malkova et al., 2012). However, not all children with congenital Rubella develop ASD. Similarly, the autistic-like symptoms in the rodent MIA model are modified by multiple environmental factors, including the gut microbiota (Hsiao et al., 2013). Further, the rodent MIA model appears rather inefficient to activate microglial or provoke synaptic deficits like those reported in ASD patients (Morgan et al., 2010; Suzuki et al., 2013; De Rubeis et al., 2014). These disparities suggest that prenatal infection and MIA may only alter the immune system in offspring, making the 
A

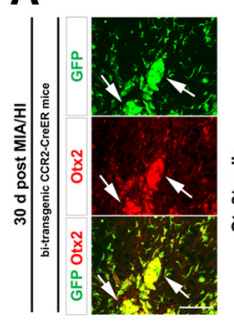

D

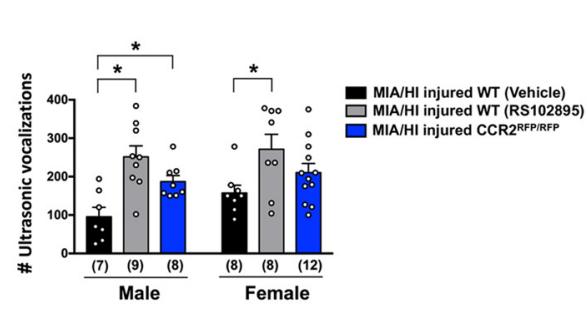

F

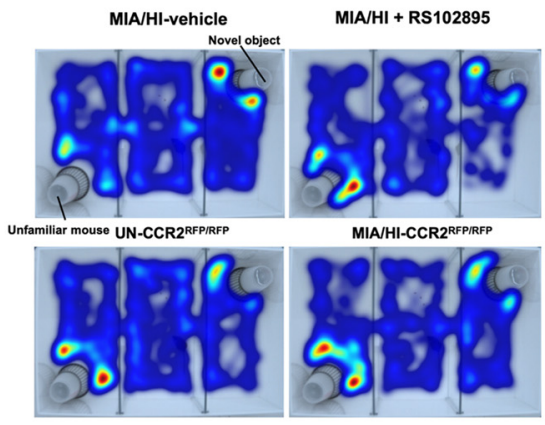

B

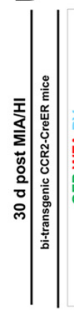

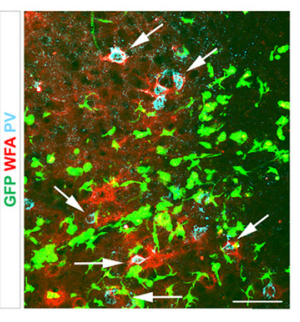

C

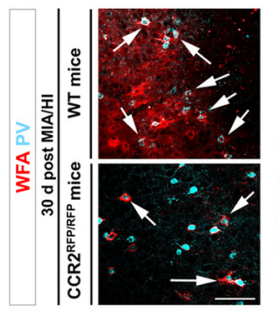

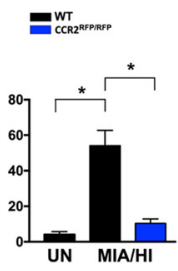

E
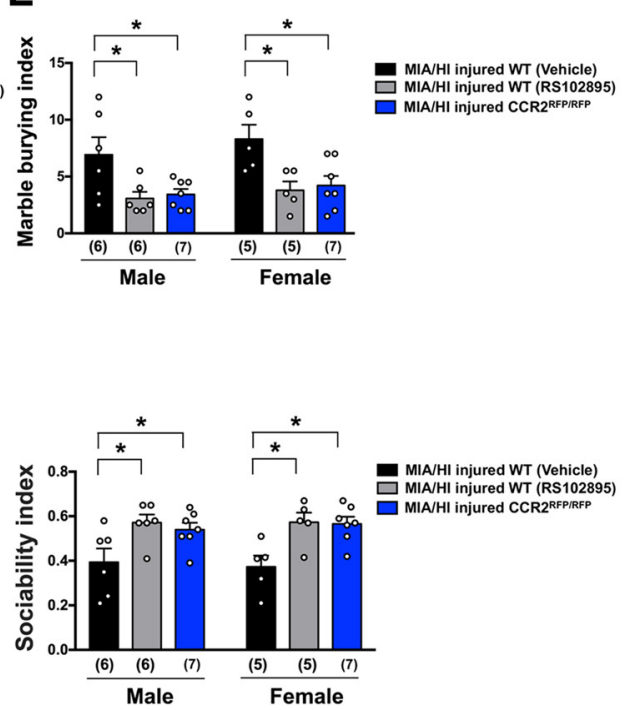

G

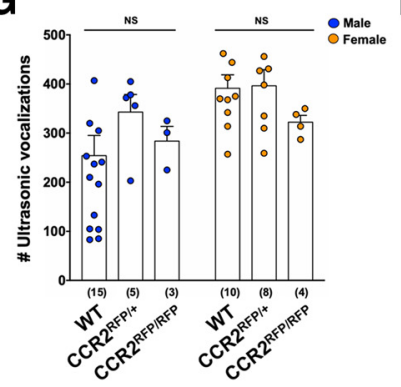

H
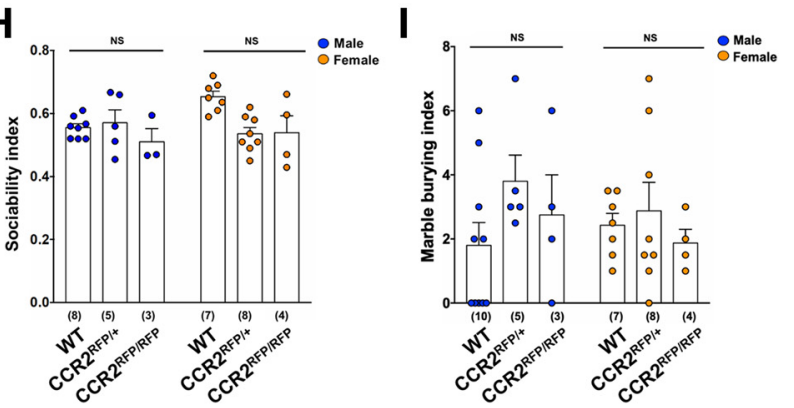

Figure 7. CCR2-targeted intervention reduces PNN and autistic-like behaviors after MIA/HI. $A$, Immunostaining showed that many GFP ${ }^{+}$monocyte derivatives continued to express 0 tx2 at $30 \mathrm{~d}$ after MIA/HI in CCR2-CreER(T2); R26R-CAG-ZSGreen (Ai6) mice. The dual-MIA/HI insult also markedly increased the number of 0tx2 ${ }^{+}$cells in the hippocampal CA3 sector at $30 \mathrm{~d}$ after MIA/ HI $(n=3)$. Scale bar, $100 \mu \mathrm{m}$. Data are mean \pm SEM. $* p<0.05$ (Student's $t$ test). $B$, Immunostaining showed that WFA-wrapped PV ${ }^{+}$neurons were often surrounded by GFP ${ }^{+}$monocyte derivatives in the hippocampus at $30 \mathrm{~d}$ after MIA/HI in CCR2-CreER(T2); R26R-CAG-ZsGreen (Ai6) mice $(n=3)$. Scale bar, $100 \mu \mathrm{m}$. C, CCR2 ${ }^{\text {RFPRFPP }}$ mice showed significantly fewer WFA-wrapped $\mathrm{PV}^{+}$neurons in the hippocampal CA3 sector than WT mice at $30 \mathrm{~d}$ after MIA/HI ( $n=4$ for each group). Scale bar, $100 \mu \mathrm{m}$. Data are mean \pm SEM. $* p<0.05$ (Kruskal-Wallis test followed by Dunn's multiple comparison test). $\mathbf{D}-\boldsymbol{F}$, RS102895 treatment and CCR2 deletion (in CCR2 ${ }^{\text {RFP/RFP }}$ mice) significantly reduced MIA/HI-induced aberrations in USV (D), stereotyped marble-burying behaviors $(\boldsymbol{E})$, and social preference index $(\boldsymbol{F}, \boldsymbol{G})$ in mice of both sexes at 2 months of age, except for female $C\left(R 2^{R F P / R F P}\right.$ mice in USV. Control experiments showed that unchallenged WT, $C\left(R 2^{R F P /+}\right.$, and $C\left(R 2^{R F P / R F P}\right.$ mice showed no difference in USV, sociability, and repetitive behaviors at 2 months of age $G-I$. G-I, CCR2 deficiency did not cause autistic-like behaviors. WT, $C\left(R 2^{R F P /+}\right.$, and $C\left(R 2^{R F P / R F P}\right.$ mice showed comparable performance in USV $(\boldsymbol{G})$, social interaction preference $(\boldsymbol{H})$, and stereotyped marble-burying behaviors $(\boldsymbol{I})$. The sample size for each group was labeled. Data are mean \pm SEM for both sexes. $* p<0.05$ (two-way ANOVA and Tukey's multiple comparisons post hoc test). NS: not significant.

offspring brain more sensitive to an array of secondary hits, including neonatal HI (Badawi et al., 2006; van Handel et al., 2007; Getahun et al., 2017).

In this study, we combined prenatal poly(I:C)-induced MIA and cerebral $\mathrm{HI}$ at postnatal day 10 to form a novel, two-hit MIA/HI model. Previous study has shown that poly(I:C) pretreatment at $14 \mathrm{~h}$ before $\mathrm{HI}$ increased neonatal damage brain (Stridh et al., 2013), but to our knowledge, the present study is the first to demonstrate sensitization effects by prenatal injection of poly(I:C) on neonatal HI insult in the offspring. Our results confirmed that MIA per se is a feeble stimulus of microglia in the offspring (Smolders et al., 2015; Bergdolt and Dunaevsky, 2019), but it skews the offspring toward intensified immune responses to a secondary $\mathrm{HI}$ insult. Conversely, neonatal $\mathrm{HI}$ by itself fails to elicit autistic-like symptoms, nor did it conceal the aberrant behaviors in MIA offspring. Rather, the combined MIA/HI insult produces autistic-like behaviors with additional features, including monocytic infiltrates, amoeboid microglial clusters, increased PNNs, plus reduced synaptic density and the PSD-95, Homer-1, and MeCP2 expression (Figs. 2-4). Notably, 


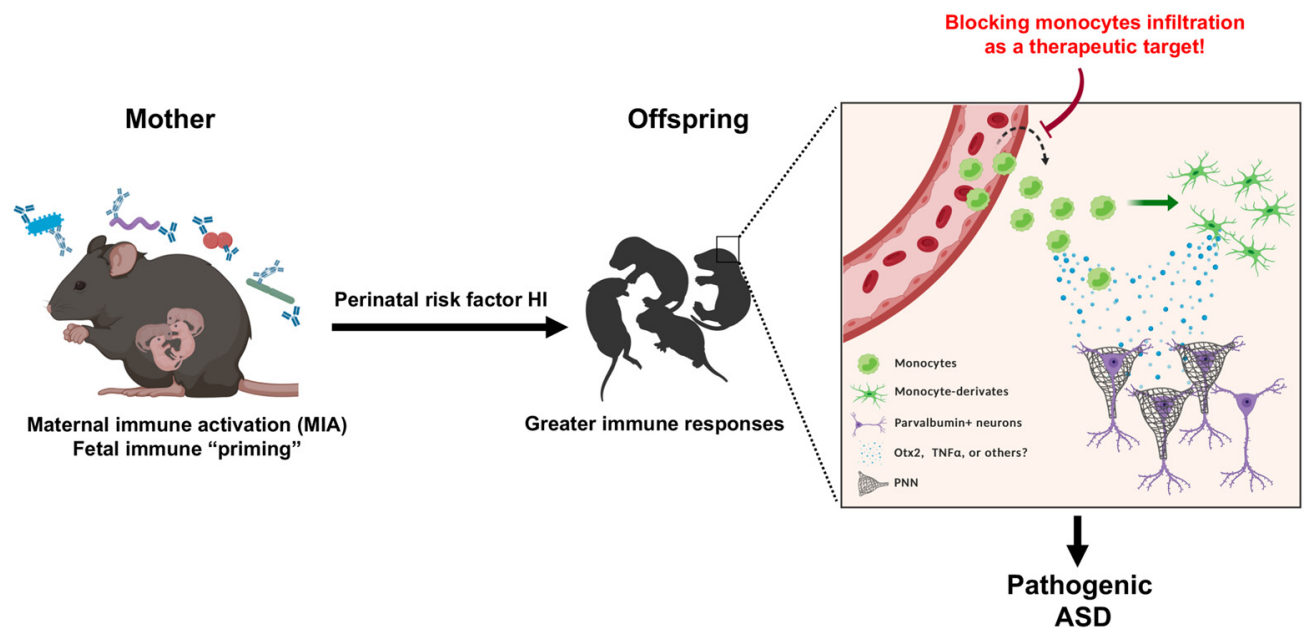

Figure 8. Mechanism by which monocytes may induce autistic-like behaviors after MIA/HI. We hypothesize that prenatal infection/MIA (first-hit) sensitizes monocytes to infiltrate into the offspring brain after perinatal HI injury (second-hit). Infiltrating monocytes and their derivatives produce 0tx2 and proinflammatory cytokines (e.g., TNF $\alpha$ ) to promote PNNs and synaptic elimination, respectively, leading to impaired neural network and autistic-like behaviors (created with BioRender).

microglia activation and loss-of-function mutations of PSD-95, Homer-1, and MeCP2 are detected in the ASD or Rett syndrome patients (Morgan et al., 2010; Tsai et al., 2012; Suzuki et al., 2013; De Rubeis et al., 2014; Lelieveld et al., 2016). Whether ASD patients have a reduced synaptic density is less certain, but synaptic dysfunction and elimination are often encountered in neuropsychiatric disorders (Sekar et al., 2016; Martínez-Cerdeño, 2017; Lima Caldeira et al., 2019). Forced expression of autismrelated Shank3 also stimulates functional dendritic spine synapses (Roussignol et al., 2005). Hence, the two-hit MIA/HI model appears to capture a broader spectrum of ASD aberrations than the standard MIA model. Future studies are warranted to test whether the combination of autism-related mutations with neonatal HI also enhance the ASD-specific behavioral and pathologic aberrations in mice.

Are these additional ASD-like features after MIA/HI insult entirely because of increased brain injury? Two lines of reasoning suggest against this scenario. First, although the MIA/HI-injured offspring showed reduced expression of postsynaptic PSD-95 and Homer-1, plus MecP2, in the hippocampus, the amount of presynaptic synaptotagmin protein was not lessened, as would be expected by greater brain damage. Rather, our results resonate with the preferential postsynaptic deficits in ASD, as shown by human genetic studies and microglia-mediated synaptic elimination in animal model (Schafer et al., 2012; De Rubeis et al., 2014; Wu et al., 2015). Second, although 10\% oxygen was used for hypoxia in our study to minimize the HI and MIA/HI insult, previous studies using more severe hypoxia ( $8 \%$ oxygen) did not report autistic-like behaviors in injured murine neonates (Ten et al., 2004; Diaz et al., 2017), suggesting that severe HI brain damage per se does not guarantee ASD-like symptoms. Nevertheless, future studies are warranted to test whether mice subjected to acute LPS- or poly(I:C)-sensitized HI injury develop social interaction deficits (Eklind et al., 2005; Stridh et al., 2013; Yang et al., 2013).

\section{Monocytes in the two-hit MIA/HI model of neurodevelopmental defects}

Regarding the pathologic mechanisms of MIA, a commonly held view posits that MIA induces maternal effectors, such as IL-17 $\alpha$ or IL-6, to directly attack the fetal brains, although permanent immune dysregulation in adult offspring was also reported
(Smith et al., 2007; Hsiao et al., 2012; Choi et al., 2016). A recent study indicates that the peripheral inflammatory stimuli in adult mice induce long-term immune memory in the microglia via epigenetic reprogramming (Wendeln et al., 2018). Thus, prenatal MIA may modify the "responsiveness" of monocytes or other immune cells to a secondary neonatal HI insult in offspring. Consistent with this hypothesis, neither MIA nor pure-HI elicits robust $\mathrm{NF} \kappa \mathrm{B}$ signaling in neonatal brains, but the combination of both insults exerts synergistic effects and is particularly potent in attracting the influx of monocytes and the formation of PNNs while reducing the synaptic density. Moreover, genetic or pharmacological intervention of the MCP1-CCR2 chemoattractant pathway significantly reduced autistic-like behaviors and WFAenwrapped PV neurons in the MIA/HI-injured mice (Fig. 7). These results suggest important functions of monocytes in the two-hit model of neurodevelopmental defects (Fig. 8).

Our results also suggested several mechanisms by which monocytes may induce autistic-like behaviors. First, because invading Ly6 $\mathrm{C}^{\text {hi }}$ monocytes are known to secrete proinflammatory cytokines and interact with the resident microglia to cause tissue injury (Geissmann et al., 2003; Murray, 2018), the greater influx of monocyte in post-MIA/HI brains may augment structural destruction to perturb neurodevelopment. Second, while peripheral monocytes have a short half-life in the blood, our fate-mapping experiment using CCR2-CreER mice showed that monocyte derivatives can survive for up to $30 \mathrm{~d}$ and express TNF $\alpha$ in post-MIA/HI brains (Figs. 5, 6). The expression of Trem 2 was also increased in the microglia and $\mathrm{Mo} / \mathrm{M} \varphi$ after dual MIA/HI insult (Fig. 3). Because both TNF $\alpha$ and Trem2 have been implicated in microglia-mediated synaptic elimination, their continuous expression by monocyte derivatives after MIA/HI brain injury may facilitate synaptic impairment (Schafer et al., 2012; Wu et al., 2015). Third, our results showed that the infiltrating monocytes expressed Otx2, a homeoprotein and the key regulator of PNN formation (Fawcett et al., 2019), correlated with autistic-like behaviors in post-MIA/HI offspring (Figs. 4, 7). These results suggest that monocytic infiltrates may provide an ectopic source of Otx2 to promote PNNs and interfere with the development of neural network.

In conclusion, our study introduces a novel two-hit model of neurodevelopmental defects and suggests the contribution by monocytes to the onset of autistic-like behaviors after combined 
MIA/HI. Limited by its scope, the present study has not defined the nature of immune priming of monocytes in the MIA offspring. Future studies are warranted to determine whether the "MIA-mediated priming" involves epigenetic reprogramming of monocytes or accelerated maturation of the immune system as a whole. Finally, examining the effects of monocyte-specific deletion of Otx 2 or TNF $\alpha$ might help to unravel the molecular underpinnings of autistic-like behaviors.

\section{References}

Adhami F, Yu D, Yin W, Schloemer A, Burns KA, Liao G, Degen JL, Chen J, Kuan CY (2008) Deleterious effects of plasminogen activators in neonatal cerebral hypoxia ischemia. Am J Pathol 172:1704-1716.

American Psychiatric Association (2013) Diagnostic and statistical manual of mental disorders, Ed 5. Washington, DC: American Psychiatric Association.

Badawi N, Dixon G, Felix JF, Keogh JM, Petterson B, Stanley FJ, Kurinczuk JJ (2006) Autism following a history of newborn encephalopathy: more than a coincidence? Dev Med Child Neurol 48:85-89.

Bennett ML, Bennett FC, Liddelow SA, Ajami B, Zamanian JL, Fernhoff NB, Mulinyawe SB, Bohlen CJ, Adil A, Tucker A, Weissman IL, Chang EF, Li G, Grant GA, Hayden Gephart MG, Barres BA (2016) New tools for studying microglia in the mouse and human CNS. Proc Natl Acad Sci USA 113:E1738-E1746.

Bergdolt L, Dunaevsky A (2019) Brain changes in a maternal immune activation model of neurodevelopmental brain disorders. Prog Neurobiol 175:1-19.

Chen HR, Sun YY, Chen CW, Kuo YM, Kuan IS, Li ZR, Short-Miller JC, Smucker MR, Kuan CY (2020) Fate-mapping via CCR2CreER mice reveals monocyte-to-microglia transition in development and neonatal stroke. Sci Adv 6:eabb2119.

Choi GB, Yim YS, Wong H, Kim S, Kim H, Kim SV, Hoeffer CA, Littman DR, Huh JR (2016) The maternal interleukin-17a pathway in mice promotes autism-like phenotypes in offspring. Science 351:933-939.

Coiro P, Padmashri R, Suresh A, Spartz E, Pendyala G, Chou S, Jung Y, Meays B, Roy S, Gautam N, Alnouti Y, Li M, Dunaevsky A (2015) Impaired synaptic development in a maternal immune activation mouse model of neurodevelopmental disorders. Brain Behav Immun 50:249258.

Cronk JC, Filiano AJ, Louveau A, Marin I, Marsh R, Ji E, Goldman DH, Smirnov I, Geraci N, Acton S, Overall CC, Kipnis J (2018) Peripherally derived macrophages can engraft the brain independent of irradiation and maintain an identity distinct from microglia. J Exp Med 215:16271647.

De Rubeis S, He X, Goldberg AP, Poultney CS, Samocha K, Ercument Cicek A, Kou Y, Liu L, Fromer M, Walker S, Singh T, Klei L, Kosmicki J, Fu SC, Aleksic B, Biscaldi M, Bolton PF, Brownfeld JM, Cai J, Campbell NG, et al. (2014) Synaptic, transcriptional and chromatin genes disrupted in autism. Nature 515:209-215.

Diaz J, Abiola S, Kim N, Avaritt O, Flock D, Yu J, Northington FJ, ChavezValdez R (2017) Therapeutic hypothermia provides variable protection against behavioral deficits after neonatal hypoxia ischemia: a potential role for brain-derived neurotrophic factor. Dev Neurosci 39:257-272.

DiStasio MM, Nagakura I, Nadler MJ, Anderson MP (2019) T lymphocytes and cytotoxic astrocyte blebs correlate across autism brains. Ann Neurol 86:885-898.

Djukic M, Mildner A, Schmidt H, Czesnik D, Bruck W, Priller J, Nau R, Prinz M (2006) Circulating monocytes engraft in the brain, differentiate into microglia and contribute to the pathology following meningitis in mice. Brain 129:2394-2403.

Eklind S, Mallard C, Arvidsson P, Hagberg H (2005) Lipopolysaccharide induces both a primary and a secondary phase of sensitization in the developing rat brain. Pediatr Res 58:112-116.

Estes ML, McAllister AK (2016) Maternal immune activation: implications for neuropsychiatric disorders. Science 353:772-777.

Fawcett JW, Oohashi T, Pizzorusso T (2019) The roles of perineuronal nets and the perinodal extracellular matrix in neuronal function. Nat Rev Neurosci 20:451-465.

Filipello F, Morini R, Corradini I, Zerbi V, Canzi A, Michalski B, Erreni M, Markicevic M, Starvaggi-Cucuzza C, Otero K, Piccio L, Cignarella F,
Perrucci F, Tamborini M, Genua M, Rajendran L, Menna E, Vetrano S, Fahnestock M, Paolicelli RC, et al. (2018) The microglial innate immune receptor TREM2 is required for synapse elimination and normal brain connectivity. Immunity 48:979-991.e978.

Folstein S, Rutter M (1977) Genetic influences and infantile autism. Nature 265:726-728.

Geissmann F, Jung S, Littman DR (2003) Blood monocytes consist of two principal subsets with distinct migratory properties. Immunity 19:71-82.

Geschwind DH (2009) Advances in autism. Annu Rev Med 60:367-380.

Getahun D, Fassett MJ, Peltier MR, Wing DA, Xiang AH, Chiu V, Jacobsen SJ (2017) Association of perinatal risk factors with autism spectrum disorder. Am J Perinatol 34:295-304.

Giovanoli S, Engler H, Engler A, Richetto J, Voget M, Willi R, Winter C, Riva MA, Mortensen PB, Feldon J, Schedlowski M, Meyer U (2013) Stress in puberty unmasks latent neuropathological consequences of prenatal immune activation in mice. Science 339:1095-1099.

Heiman M, Kulicke R, Fenster RJ, Greengard P, Heintz N (2014) Cell typespecific mRNA purification by translating ribosome affinity purification (TRAP). Nat Protoc 9:1282-1291.

Hsiao EY, McBride SW, Chow J, Mazmanian SK, Patterson PH (2012) Modeling an autism risk factor in mice leads to permanent immune dysregulation. Proc Natl Acad Sci USA 109:12776-12781.

Hsiao EY, McBride SW, Hsien S, Sharon G, Hyde ER, McCue T, Codelli JA, Chow J, Reisman SE, Petrosino JF, Patterson PH, Mazmanian SK (2013) Microbiota modulate behavioral and physiological abnormalities associated with neurodevelopmental disorders. Cell 155:1451-1463.

Jung S, Aliberti J, Graemmel P, Sunshine MJ, Kreutzberg GW, Sher A, Littman DR (2000) Analysis of fractalkine receptor CX(3)CR1 function by targeted deletion and green fluorescent protein reporter gene insertion. Mol Cell Biol 20:4106-4114.

Krishnan K, Wang BS, Lu J, Wang L, Maffei A, Cang J, Huang ZJ (2015) $\mathrm{MeCP} 2$ regulates the timing of critical period plasticity that shapes functional connectivity in primary visual cortex. Proc Natl Acad Sci USA 112: E4782-E4791.

Lammert CR, Lukens JR (2019) Modeling autism-related disorders in mice with maternal immune activation (MIA). Methods Mol Biol 1960:227236.

Lelieveld SH, Reijnders MR, Pfundt R, Yntema HG, Kamsteeg EJ, de Vries P, de Vries BB, Willemsen MH, Kleefstra T, Löhner K, Vreeburg M, Stevens SJ, van der Burgt I, Bongers EM, Stegmann AP, Rump P, Rinne T, Nelen MR, Veltman JA, Vissers LE, et al. (2016) Meta-analysis of 2,104 trios provides support for 10 new genes for intellectual disability. Nat Neurosci 19:1194-1196.

Lima Caldeira G, Peca J, Carvalho AL (2019) New insights on synaptic dysfunction in neuropsychiatric disorders. Curr Opin Neurobiol 57:62-70.

Lipina TV, Zai C, Hlousek D, Roder JC, Wong AH (2013) Maternal immune activation during gestation interacts with Discl point mutation to exacerbate schizophrenia-related behaviors in mice. J Neurosci 33:7654-7666.

Malkova NV, Yu CZ, Hsiao EY, Moore MJ, Patterson PH (2012) Maternal immune activation yields offspring displaying mouse versions of the three core symptoms of autism. Brain Behav Immun 26:607-616.

Martínez-Cerdeño V (2017) Dendrite and spine modifications in autism and related neurodevelopmental disorders in patients and animal models. Dev Neurobiol 77:393-404.

Meyer U (2014) Prenatal poly(I:C) exposure and other developmental immune activation models in rodent systems. Biol Psychiatry 75:307315.

Mirzadegan T, Diehl F, Ebi B, Bhakta S, Polsky I, McCarley D, Mulkins M, Weatherhead GS, Lapierre JM, Dankwardt J, Morgans D, Wilhelm R, Jarnagin K (2000) Identification of the binding site for a novel class of CCR2b chemokine receptor antagonists: binding to a common chemokine receptor motif within the helical bundle. J Biol Chem 275:2556225571.

Morgan JT, Chana G, Pardo CA, Achim C, Semendeferi K, Buckwalter J, Courchesne E, Everall IP (2010) Microglial activation and increased microglial density observed in the dorsolateral prefrontal cortex in autism. Biol Psychiatry 68:368-376.

Murray PJ (2018) Immune regulation by monocytes. Semin Immunol 35:1218.

Murthy S, Kane GA, Katchur NJ, Lara Mejia PS, Obiofuma G, Buschman TJ, McEwen BS, Gould E (2019) Perineuronal nets, inhibitory interneurons, 
and anxiety-related ventral hippocampal neuronal oscillations are altered by early life adversity. Biol Psychiatry 85:1011-1020.

Pietropaolo S, Guilleminot A, Martin B, D'Amato FR, Crusio WE (2011) Genetic-background modulation of core and variable autistic-like symptoms in Fmr1 knock-out mice. PLoS One 6:e17073.

Prinz M, Jung S, Priller J (2019) Microglia biology: one century of evolving concepts. Cell 179:292-311.

Rice JE 3rd, Vannucci RC, Brierley JB (1981) The influence of immaturity on hypoxic-ischemic brain damage in the rat. Ann Neurol 9:131-141.

Roussignol G, Ango F, Romorini S, Tu JC, Sala C, Worley PF, Bockaert J, Fagni L (2005) Shank expression is sufficient to induce functional dendritic spine synapses in aspiny neurons. J Neurosci 25:3560-3570.

Saederup N, Cardona AE, Croft K, Mizutani M, Cotleur AC, Tsou CL, Ransohoff RM, Charo IF (2010) Selective chemokine receptor usage by central nervous system myeloid cells in CCR2-red fluorescent protein knock-in mice. PLoS One 5:e13693.

Schafer DP, Lehrman EK, Kautzman AG, Koyama R, Mardinly AR, Yamasaki R, Ransohoff RM, Greenberg ME, Barres BA, Stevens B (2012) Microglia sculpt postnatal neural circuits in an activity and complementdependent manner. Neuron 74:691-705.

Sekar A, Bialas AR, de Rivera H, Davis A, Hammond TR, Kamitaki N, Tooley K, Presumey J, Baum M, Van Doren V, Genovese G, Rose SA, Handsaker RE, Daly MJ, Carroll MC, Stevens B, McCarroll SA, Schizophrenia Working Group of the Psychiatric Genomics Consortium (2016) Schizophrenia risk from complex variation of complement component 4. Nature 530:177-183.

Servadio M, Vanderschuren LJ, Trezza V (2015) Modeling autism-relevant behavioral phenotypes in rats and mice: do 'autistic' rodents exist? Behav Pharmacol 26:522-540.

Smith SE, Li J, Garbett K, Mirnics K, Patterson PH (2007) Maternal immune activation alters fetal brain development through interleukin-6. J Neurosci 27:10695-10702.

Smolders S, Smolders SM, Swinnen N, Gartner A, Rigo JM, Legendre P, Brone B (2015) Maternal immune activation evoked by polyinosinic:polycytidylic acid does not evoke microglial cell activation in the embryo. Front Cell Neurosci 9:301.
Spatazza J, Lee HH, Di Nardo AA, Tibaldi L, Joliot A, Hensch TK, Prochiantz A (2013) Choroid-plexus-derived Otx2 homeoprotein constrains adult cortical plasticity. Cell Rep 3:1815-1823.

Stridh L, Mottahedin A, Johansson ME, Valdez RC, Northington F, Wang X, Mallard C (2013) Toll-like receptor-3 activation increases the vulnerability of the neonatal brain to hypoxia ischemia. J Neurosci 33:1204112051.

Suzuki K, Sugihara G, Ouchi Y, Nakamura K, Futatsubashi M, Takebayashi K, Yoshihara Y, Omata K, Matsumoto K, Tsuchiya KJ, Iwata Y, Tsujii M, Sugiyama T, Mori N (2013) Microglial activation in young adults with autism spectrum disorder. JAMA Psychiatry 70:49-58.

Ten VS, Wu EX, Tang H, Bradley-Moore M, Fedarau MV, Ratner VI, Stark RI, Gingrich JA, Pinsky DJ (2004) Late measures of brain injury after neonatal hypoxia ischemia in mice. Stroke 35:2183-2188.

Tsai NP, Wilkerson JR, Guo W, Maksimova MA, DeMartino GN, Cowan CW, Huber KM (2012) Multiple autism-linked genes mediate synapse elimination via proteasomal degradation of a synaptic scaffold PSD-95. Cell 151:1581-1594.

van Handel M, Swaab H, de Vries LS, Jongmans MJ (2007) Long-term cognitive and behavioral consequences of neonatal encephalopathy following perinatal asphyxia: a review. Eur J Pediatr 166:645-654.

Wang F, Flanagan J, Su N, Wang LC, Bui S, Nielson A, Wu X, Vo HT, Ma XJ, Luo Y (2012) RNAscope: a novel in situ RNA analysis platform for formalin-fixed, paraffin-embedded tissues. J Mol Diagn 14:22-29.

Wendeln AC, Degenhardt K, Kaurani L, Gertig M, Ulas T, Jain G, Wagner J, Häsler LM, Wild K, Skodras A, Blank T, Staszewski O, Datta M, Centeno TP, Capece V, Islam MR, Kerimoglu C, Staufenbiel M, Schultze JL, Beyer $\mathrm{M}$, et al. (2018) Innate immune memory in the brain shapes neurological disease hallmarks. Nature 556:332-338.

Wu Y, Dissing-Olesen L, MacVicar BA, Stevens B (2015) Microglia: dynamic mediators of synapse development and plasticity. Trends Immunol 36:605-613.

Yang D, Sun YY, Nemkul N, Baumann JM, Shereen A, Dunn RS, Wills-Karp M, Lawrence DA, Lindquist DM, Kuan CY (2013) Plasminogen activator inhibitor-1 mitigates brain injury in a rat model of infection-sensitized neonatal hypoxia ischemia. Cereb Cortex 23:1218-1229. 